\title{
CONFLICT FREE COLORINGS OF (STRONGLY) ALMOST DISJOINT SET-SYSTEMS
}

\author{
ANDRÁS HAJNAL, ISTVÁN JUHÁSZ, LAJOS SOUKUP, \\ AND ZOLTÁN SZENTMIKLÓSSY
}

\begin{abstract}
A} \rightarrow \rho$ is called a conflict free coloring of the set-system $\mathcal{A}$ (with $\rho$ colors) if$$
\forall A \in \mathcal{A} \exists \zeta<\rho\left(\left|A \cap f^{-1}\{\zeta\}\right|=1\right) .
$$

The conflict free chromatic number $\chi_{\mathrm{CF}}(\mathcal{A})$ of $\mathcal{A}$ is the smallest $\rho$ for which $\mathcal{A}$ admits a conflict free coloring with $\rho$ colors.

$\mathcal{A}$ is a $(\lambda, \kappa, \mu)$-system if $|\mathcal{A}|=\lambda,|A|=\kappa$ for all $A \in \mathcal{A}$, and $\mathcal{A}$ is $\mu$-almost disjoint, i.e. $\left|A \cap A^{\prime}\right|<\mu$ for distinct $A, A^{\prime} \in \mathcal{A}$. Our aim here is to study

$$
\chi_{\mathrm{CF}}(\lambda, \kappa, \mu)=\sup \left\{\chi_{\mathrm{CF}}(\mathcal{A}): \mathcal{A} \text { is a }(\lambda, \kappa, \mu) \text {-system }\right\}
$$

for $\lambda \geq \kappa \geq \mu$, actually restricting ourselves to $\lambda \geq \omega$ and $\mu \leq \omega$.

For instance, we prove that

- for any limit cardinal $\kappa$ (or $\kappa=\omega$ ) and integers $n \geq 0, k>0, \mathrm{GCH}$ implies

$$
\chi_{\mathrm{CF}}\left(\kappa^{+n}, t, k+1\right)=\left\{\begin{array}{rr}
\kappa^{+(n+1-i)} & \text { if } i \cdot k<t \leq(i+1) \cdot k \\
& i=1, \ldots, n \\
\kappa & \text { if }(n+1) \cdot k<t ;
\end{array}\right.
$$

- if $\lambda \geq \kappa \geq \omega>d>1$, then $\lambda<\kappa^{+\omega}$ implies $\chi_{\mathrm{CF}}(\lambda, \kappa, d)<\omega$ and $\lambda \geq \beth_{\omega}(\kappa)$ implies $\chi_{\mathrm{CF}}(\lambda, \kappa, d)=\omega$;

- GCH implies $\chi_{\mathrm{CF}}(\lambda, \kappa, \omega) \leq \omega_{2}$ for $\lambda \geq \kappa \geq \omega_{2}$ and $\mathrm{V}=\mathrm{L}$ implies $\chi_{\mathrm{CF}}(\lambda, \kappa, \omega) \leq \omega_{1}$ for $\lambda \geq \kappa \geq \omega_{1}$;

- the existence of a supercompact cardinal implies the consistency of GCH plus $\chi_{\mathrm{CF}}\left(\aleph_{\omega+1}, \omega_{1}, \omega\right)=\aleph_{\omega+1}$ and $\chi_{\mathrm{CF}}\left(\aleph_{\omega+1}, \omega_{n}, \omega\right)=\omega_{2}$ for $2 \leq n \leq \omega$;

- CH implies $\chi_{\mathrm{CF}}\left(\omega_{1}, \omega, \omega\right)=\chi_{\mathrm{CF}}\left(\omega_{1}, \omega_{1}, \omega\right)=\omega_{1}$, while $M A_{\omega_{1}}$ implies $\chi_{\mathrm{CF}}\left(\omega_{1}, \omega, \omega\right)=\chi_{\mathrm{CF}}\left(\omega_{1}, \omega_{1}, \omega\right)=\omega$.

Date: March, 2010.

2000 Mathematics Subject Classification. 03E35, 03E05.

Key words and phrases. coloring, conflict-free coloring, almost disjoint, essentially disjoint.

The preparation of this paper was partially supported by OTKA grants K 61600 and K 68262 . 


\section{INTRODUCTION}

If $\mathcal{A}$ is a set-system and $\rho$ is a cardinal then a function $f: \cup \mathcal{A} \rightarrow \rho$ is called a proper coloring of $\mathcal{A}$ with $\rho$ colors if $f$ takes at least 2 values on each $A \in \mathcal{A}$. The smallest $\rho$ for which $\mathcal{A}$ admits a proper coloring with $\rho$ colors is the chromatic number of $\mathcal{A}$ and is denoted by $\chi(\mathcal{A})$. The chromatic numbers of various set-systems, in particular almost disjoint ones, had been systematically studied by Erdős and Hajnal and others in [3], [4], and [5].

A function $f: \cup \mathcal{A} \rightarrow \rho$ is called a conflict free coloring of $\mathcal{A}$ with $\rho$ colors if

$$
\forall A \in \mathcal{A} \exists \zeta<\rho\left(\left|A \cap f^{-1}\{\zeta\}\right|=1\right) .
$$

We say that $f$ is a weak conflict free coloring of $\mathcal{A}$ if in the above definition the assumption $\operatorname{dom}(f)=\cup \mathcal{A}$ is weakened to $\operatorname{dom}(f) \subset \cup \mathcal{A}$.

The conflict-free chromatic number and the weak conflict-free chromatic number of a set-system $\mathcal{A}$, denoted by

$$
\chi_{\mathrm{CF}}(\mathcal{A}) \text { and } w \chi_{\mathrm{CF}}(\mathcal{A})
$$

respectively, are defined as the minimum number of colors needed for a conflict free or a weak conflict-free coloring of $\mathcal{A}$, respectively.

Conflict-free colorings of hypergraphs, that is of systems of finite sets, were first studied in Cheilaris [1] and Pach-Tardos [13]. Earlier, conflict-free colorings were mainly considered for some concrete hypergraphs, usually defined by geometric means [6]. János Pach suggested to us that it would be worth while to study the conflict free colorings of almost disjoint transfinite set systems. It took little time to convince us.

Before going on with the story we state a few very elementary facts. Note first that $\chi(\mathcal{A})$ is only defined if every member of $\mathcal{A}$ has at least two elements, so from here on this is assumed for every set-system $\mathcal{A}$.

Proposition 1.1. (1) $\chi(\mathcal{A}) \leq \chi_{\mathrm{CF}}(\mathcal{A}) \leq w \chi_{\mathrm{CF}}(\mathcal{A})+1$.

(2) $\chi(\mathcal{A})=\chi_{\mathrm{CF}}(\mathcal{A})$ provided $|A| \leq 3$ for all $A \in \mathcal{A}$.

(3) For each $\kappa \geq \omega$ there exists a quadruple system $\mathcal{A}$ with $\chi(\mathcal{A})=2$ and $\chi_{\mathrm{CF}}(\mathcal{A})=\kappa$.

Proof. The first statement is trivial, the second follows from $2+2>3$. To see the third, let

$$
\mathcal{A}=\left\{H \in[\kappa]^{4}: H \text { contains two even and two odd ordinals }\right\} .
$$


For any cardinals $\mu$ and $\nu$, the set system $\mathcal{A}$ is called $(\mu, \nu)$-almost disjoint if

$$
|\cap \mathcal{B}|<\mu
$$

whenever $\mathcal{B} \in[\mathcal{A}]^{\nu}$. We simply write $\mu$-almost disjoint instead of $(\mu, 2)$-almost disjoint.

A graph $G=\langle V, E\rangle$ is called $(\mu, \nu)$-almost disjoint iff the family $\{E(v): v \in V\}$ is $(\mu, \nu)$-almost disjoint, where $E(v)=\{w \in V$ : $\{v, w\} \in E\}$. In [4], Erdös and Hajnal proved, in 1966, that if $n<$ $\omega$ and $G$ is an $\left(n, \omega_{1}\right)$-almost disjoint graph, then $\chi(G) \leq \omega$, which of course means $\chi(E) \leq \omega$. They tried to state a generalization of this result for set-systems consisting of finite sets, but failed. Such a generalization was found in the triple paper [5] with B.Rothchild, where some results were proved for finitary $(\mu, \nu)$-almost disjoint set-systems. In Part I we prove results for such set-systems that are improvements of the results of [5]. The work started in [5] was continued in the almost ninety page long triple paper [2] of Erdős, Galvin and Hajnal. Although we could find some improvements of the results of this paper as well, we did not dare to start to investigate this methodically.

Our main objects of study will be the (weak) conflict free chromatic numbers of $(\lambda, \kappa, \mu)$-systems: $\mathcal{A}$ is a $(\lambda, \kappa, \mu)$-system if $|\mathcal{A}|=\lambda,|A|=\kappa$ for all $A \in \mathcal{A}$, and $\mathcal{A}$ is $\mu$-almost disjoint. We shall always assume that $\lambda \geq \kappa \geq \mu$ and that $\lambda$ is infinite. These assumptions imply that if $\mathcal{A}$ is a $(\lambda, \kappa, \mu)$-system then $|\cup \mathcal{A}| \leq \lambda$, hence $\mathcal{A}$ has an isomorphic copy $\mathcal{B} \subset[\lambda]^{\kappa}$. Conversely, if $\mu<\omega$ then for every $\mu$-almost disjoint $\mathcal{A} \subset[\lambda]^{\kappa}$ we have $|\mathcal{A}| \leq \lambda$.

Now, our basic definition is the following. Let $\psi$ be any one of the functions $\chi, \chi_{\mathrm{CF}}$, or $w \chi_{\mathrm{CF}}$.

Definition 1.2. For $\lambda \geq \kappa \geq \mu$ we set

$$
\psi(\lambda, \kappa, \mu)=\sup \{\psi(\mathcal{A}): \mathcal{A} \text { is a }(\lambda, \kappa, \mu) \text {-system }\} .
$$

Let us point out certain basic properties of these. First, it is obvious that $\chi(\lambda, \kappa, \mu) \leq \chi_{\mathrm{CF}}(\lambda, \kappa, \mu)$ and

$$
w \chi_{\mathrm{CF}}(\lambda, \kappa, \mu) \leq \chi_{\mathrm{CF}}(\lambda, \kappa, \mu) \leq w \chi_{\mathrm{CF}}(\lambda, \kappa, \mu)+1 .
$$

Thus, although in some cases $w \chi_{\mathrm{CF}}(\lambda, \kappa, \mu)$ is much easier to handle than $\chi_{\mathrm{CF}}(\lambda, \kappa, \mu)$, the results on the former reveal a lot of information about the latter. Second, it is immediate from their definitions that they are monotone increasing in their first and third variables.

Intuitively, it also seems plausible that they are monotone decreasing in their second variable: the larger the sets, the more room we have to color them appropriately. For $\chi(\lambda, \kappa, \mu)$ this is obvious and all our 
results confirm this for the other two as well. Alas, we do not have a formal proof of this, so we propose it as a conjecture.

Conjecture . If $\lambda \geq \kappa>\kappa^{\prime} \geq \mu$ with $\lambda$ infinite, then

$$
\chi_{\mathrm{CF}}(\lambda, \kappa, \mu) \leq \chi_{\mathrm{CF}}\left(\lambda, \kappa^{\prime}, \mu\right) .
$$

Third, we note that if $\mu=1$, i.e. we deal with disjoint systems, then trivially $w \chi_{\mathrm{CF}}(\lambda, \kappa, 1)=1$ and $\chi(\lambda, \kappa, 1)=\chi_{\mathrm{CF}}(\lambda, \kappa, 1)=2$. Consequently, in what follows we always assume $\mu \geq 2$.

While working on this paper we found it useful to write $[\lambda, \kappa, \mu] \rightarrow \rho$ for the relation $\chi_{\mathrm{CF}}(\lambda, \kappa, \mu) \leq \rho$ and, analogously, $[\lambda, \kappa, \mu] \rightarrow_{w} \rho$ for the relation $w \chi_{\mathrm{CF}}(\lambda, \kappa, \mu) \leq \rho$.

On one hand, the behavior of these symbols shows much similarity to the symbol $M(\lambda, \kappa, \mu) \rightarrow B(\rho)$, investigated in [3], [7], and [8], meaning that every $(\lambda, \kappa, \mu)$-system has a $\rho$-transversal, i.e. a set $B$ that meets every element of $\mathcal{A}$ in a non-empty set of size $<\rho$. But the main reason for this apparent duplication of our notation is that certain variations of these arrow relations will turn out to be quite useful later.

The paper is naturally divided into three parts as follows:

Part I. $\lambda \geq \omega>\kappa \geq \mu$,

Part II. $\lambda \geq \kappa \geq \omega>\mu$,

Part III. $\lambda \geq \kappa \geq \omega=\mu$,

and the three parts are largely independent of each other. However closure arguments, in the "modern" disguise of elementary chains, have been extensively used in all three parts. This method was developed in the papers [12, 3, 7, 8], the earlier ones naturally using different terminology.

The main result of Part I is theorem 3.9 that gives a full description of $\chi_{\mathrm{CF}}(\lambda, \kappa, \mu)$ for this case in which $\kappa$ (and hence $\mu$ ) is finite. We also have ZFC results, for instance corollary 3.3 that states $\chi_{\mathrm{CF}}(\lambda, 2 k, k+1)=\lambda$ for any $\lambda \geq \omega$ and $0<k<\omega$. Of course, then conjecture "would imply $\chi_{\mathrm{CF}}(\lambda, t, k+1)=\lambda$ for $k<t<2 k$ as well. In corollary 3.5 we could prove this, with some effort, for "almost all" $\lambda$, namely those that are not successors of singular cardinals.

In Part II we first show that $\chi_{\mathrm{CF}}(\lambda, \kappa, d)$ is always countable, i.e. $[\lambda, \kappa, d] \rightarrow \omega$ holds, if $\kappa \geq \omega>d$. In fact we show something stronger that involves a modified arrow relation. To get this we first need the following notation.

Definition 1.3. If $f$ is a function and $A$ is any set, we let

$$
f[A]=\{f(\alpha): \alpha \in A \cap \operatorname{dom}(f)\}
$$

and 


$$
I_{f}(A)=\left\{\xi \in \operatorname{ran}(f):\left|A \cap f^{-1}\{\xi\}\right|=1\right\} .
$$

Thus, $f$ is a weak conflict free coloring of a set system $\mathcal{A}$ exactly if $I_{f}(A) \neq \emptyset$ for all $A \in \mathcal{A}$. Keeping this in mind, we indeed define a strengthening of the relation $[\lambda, \kappa, \mu] \rightarrow \rho$ below.

Definition 1.4. Assume that $\lambda \geq \kappa \geq \rho \geq \omega$ and $\mu \leq \kappa$. Then $[\lambda, \kappa, \mu] \Rightarrow \rho$ denotes that there is a function $f: \cup \mathcal{A} \rightarrow \rho$ such that $\left|\rho \backslash I_{f}(A)\right|<\rho$ holds for all $A \in \mathcal{A}$.

What we actually prove in theorem 4.1 is $[\lambda, \kappa, d] \Rightarrow \omega$ whenever $\kappa \geq \omega>d$.

In [3] it was proved that $M\left(\kappa, \kappa^{+n}, d\right) \rightarrow B((n+1)(d-1)+2)$ and that this is best possible assuming GCH. In Sections 5, 6, and 7 of Part II we prove analogous results for our symbols. In some sense, these chapters are the heart of our present paper. The results and their proofs seem more complicated than those from Part I, and there are a number of unsolved problems left.

By theorem 5.1, if $m$ and $d$ are natural numbers and $\kappa$ is infinite, then

$$
w \chi_{\mathrm{CF}}\left(\kappa^{+m}, \kappa, d\right) \leq\left\lfloor\frac{(m+1)(d-1)+1}{2}\right\rfloor+1 .
$$

¿From the other side, theorems 6.8 and 6.4 yield

$$
w \chi_{\mathrm{CF}}\left(\beth_{m}(\kappa), \kappa, 2\right) \geq\left\lfloor\frac{m}{2}\right\rfloor+2
$$

and

$$
w \chi_{\mathrm{CF}}\left(\beth_{m}(\kappa), \kappa, 2 \ell+1\right) \geq(m+1) \cdot \ell+1,
$$

respectively. Consequently, under $\mathrm{GCH}$ we get the exact values

$$
w \chi_{\mathrm{CF}}\left(\kappa^{+m}, \kappa, 2\right)=\lfloor m / 2\rfloor+2
$$

and

$$
w \chi_{\mathrm{CF}}\left(\kappa^{+m}, \kappa, 2 \ell+1\right)=(m+1) \cdot \ell+1 .
$$

It seems to be much more challenging to find the exact values of, say, $\chi_{\mathrm{CF}}\left(\omega_{m}, \omega, d\right)$, even under $\mathrm{GCH}$ and for $d=2$. We conjecture that $\mathrm{GCH}$ implies $\chi_{\mathrm{CF}}(\lambda, \kappa, d)=w \chi_{\mathrm{CF}}(\lambda, \kappa, d)+1$, but we could not even prove that

$$
\chi_{\mathrm{CF}}\left(\omega_{m}, \omega, 2\right)=\lfloor m / 2\rfloor+3
$$

holds for each $m \in \omega$. This equality holds for $m=0,1$ in ZFC, by proposition 7.1, and for $m=3$ under GCH , by theorem 7.7. However, for $m=2$, we cannot prove even the consistency of $\chi_{\mathrm{CF}}\left(\omega_{2}, \omega, 2\right)=4$. 
In Part III we only investigate conflict free colorings of $(\lambda, \kappa, \omega)$ systems, but it is fairly clear that most of the results would generalize for arbitrary infinite cardinals $\mu$ instead of $\omega$. This practically means that we only follow in the footsteps of the triple paper [7], leaving the cases covered only in [8] alone. Results for these cases are reserved for later publications or left for future generations.

By a result of Komjáth [9], we have $\chi\left(2^{\omega}, \omega, \omega\right)=\chi_{\mathrm{CF}}\left(2^{\omega}, \omega, \omega\right)=2^{\omega}$, and if $\boldsymbol{\beta}(\lambda)$ holds for a regular $\lambda$ then $\chi_{\mathrm{CF}}(\lambda, \omega, \omega)=\lambda$. So, in ZFC, we can not have any non-trivial upper bound for $\chi_{\mathrm{CF}}(\lambda, \omega, \omega)$. By theorem 10.3, $\mathrm{CH}$ implies $\chi_{\mathrm{CF}}\left(\omega_{1}, \omega_{1}, \omega\right)=\omega_{1}$, so even for uncountable $\kappa$ we expect to have only uncountable upper bounds for $\chi_{\mathrm{CF}}(\lambda, \kappa, \omega)$.

Such bounds can indeed be found, at least consistently. For instance, theorem 8.6 says that if $\mu^{\omega}=\mu$ holds for each $\mu<\lambda$ with $\operatorname{cf}(\mu)=\omega$, then we have $[\lambda, \kappa, \omega] \Rightarrow \omega_{2}$, hence $\chi_{\mathrm{CF}}(\lambda, \kappa, \omega) \leq \omega_{2}$, whenever $\omega_{2} \leq$ $\kappa \leq \lambda$. Moreover, if in addition we also assume $\square_{\mu}$ for all $\mu$ with $\omega=\operatorname{cf}(\mu)<\mu<\lambda$, then $\chi_{\mathrm{CF}}(\lambda, \kappa, \omega) \leq \omega_{1}$ whenever $\omega_{1} \leq \kappa \leq \lambda$, by theorem 8.7 .

These results are very sharp, at least modulo large cardinals. Indeed, we show in section 9 that the existence of a supercompact cardinal implies the consistency of GCH plus the following two equalities:

- $\chi_{\mathrm{CF}}\left(\omega_{\omega+1}, \omega_{1}, \omega\right)=\omega_{\omega+1}$,

- $\chi_{\mathrm{CF}}\left(\omega_{\omega+1}, \omega_{n}, \omega\right)=\omega_{2}$ for $2 \leq n \leq \omega$.

We close each Part by stating the problems that are nagging us most.

Our notation is standard, as e.g. in [1]. If $\lambda$ is an infinite cardinal then we call $a \lambda$-chain of elementary submodels a continuous sequence $\left\langle N_{\alpha}: \alpha<\lambda\right\rangle$ such that $N_{0}=\emptyset,\left\{N_{\alpha}: 1 \leq \alpha<\lambda\right\}$ are elementary submodels of $\left\langle H_{\theta}, \in\right\rangle$ for some fixed, appropriately chosen regular cardinal $\theta$, moreover $\left|N_{\alpha}\right|<\lambda, N_{\alpha} \in N_{\alpha+1}$ and $\alpha \subset N_{\alpha} \cap \lambda$ for $\alpha<\lambda$. If $\lambda=\kappa^{+}$then we also assume $\kappa \subset N_{1}$. We put $N_{0}=\emptyset$ to ensure that $\left\{N_{\alpha+1} \backslash N_{\alpha}: \alpha<\lambda\right\}$ be a partition of $\cup\left\{N_{\alpha}: \alpha<\lambda\right\}$.

PART I. The CASE $\lambda \geq \omega>\kappa \geq \mu$

\section{UPPER BOUNDS}

It is obvious that for every $\mathcal{A} \subset \mathcal{P}(\kappa)$ we have $\chi_{\mathrm{CF}}(\mathcal{A}) \leq \kappa$. Our next result shows that this inequality remains true for suitably almost disjoint families $\mathcal{A}$ of finite subsets of $\kappa^{+n}$ with $\omega>n>0$, provided that the members of $\mathcal{A}$ are large enough. 
Theorem 2.1. Let $\kappa \geq \nu \geq \omega$ where $\nu$ is assumed to be regular, moreover $n \geq 1$ and $k \geq 1$ be natural numbers. If $\mathcal{A}$ is a $(k+1, \nu)$ almost disjoint subfamily of $\left[\kappa^{+n-1}\right]<\omega$ such that $|A|>n \cdot k$ for every $A \in \mathcal{A}$, then $\chi_{\mathrm{CF}}(\mathcal{A}) \leq \kappa$.

Proof. We actually prove the following stronger statement $(*)_{n}$ by induction on $n \geq 1$, keeping all the other parameters fixed.

$(*)_{n}$ If $\mathcal{A} \subset\left[\kappa^{+n-1}\right]^{<\omega} \backslash\left[\kappa^{+n-1}\right]^{\leq n \cdot k}$ is $(k+1, \nu)$-almost disjoint and $g: \mathcal{A} \rightarrow[\kappa]^{<\nu}$ then there is a function $f: \kappa^{+n-1} \rightarrow \kappa$ such that

$$
I_{f}(A) \backslash g(A) \neq \emptyset
$$

for each $A \in \mathcal{A}$.

First step: $n=1$.

We define an injective function $f: \kappa \rightarrow \kappa$ inductively on $\xi<\kappa$. Assume that we have defined $f\lceil\xi$ and let

$$
\mathcal{A}_{\xi}=\{A \in \mathcal{A}: \xi=\max A\} .
$$

Clearly, $\left|\mathcal{A}_{\xi}\right|<\kappa$, hence $\left|\bigcup\left\{g(A): A \in \mathcal{A}_{\xi}\right\}\right|<\kappa$ as well. The second inequality uses that $\kappa$ is regular in case $\nu=\kappa$ and is trivial otherwise. Thus we may pick

$$
f(\xi) \in \kappa \backslash\left(f[\xi] \cup \bigcup\left\{g(A): A \in \mathcal{A}_{\xi}\right\}\right) .
$$

By the construction, we have $f(\max A) \in I_{f}(A) \backslash g(A)$ for all $A \in$ $\mathcal{A}$. (Of course, this construction does not make use of the almost disjointness or the largeness assumptions made on $\mathcal{A}$.)

Inductive step: $(*)_{n} \rightarrow(*)_{n+1}$.

Now we start with a $(k+1, \nu)$-almost disjoint system

$$
\mathcal{A} \subset\left[\kappa^{+n}\right]^{<\omega} \backslash\left[\kappa^{+n}\right]^{\leq(n+1) \cdot k}
$$

and a function $g: \mathcal{A} \rightarrow[\kappa]^{<\nu}$. Let us then fix a $\kappa^{+n}$-chain of elementary submodels $\left\langle N_{\alpha}: \alpha<\kappa^{+n}\right\rangle$ with $\mathcal{A}, g \in N_{1}$. For every $\alpha<\kappa^{+n}$ let $Y_{\alpha}=\kappa^{+n} \cap\left(N_{\alpha+1} \backslash N_{\alpha}\right), \mathcal{A}_{\alpha}=\mathcal{A} \cap\left(N_{\alpha+1} \backslash N_{\alpha}\right)$ and, finally, $\mathcal{A}_{\alpha}^{\prime}=$ $\left\{A \cap Y_{\alpha}: A \in \mathcal{A}_{\alpha}\right\}$. We may clearly assume that $\left|N_{\alpha+1}\right|=\left|Y_{\alpha}\right|=\kappa^{+n-1}$ for all $\alpha<\kappa^{+n}$.

For every $A \in \mathcal{A} \backslash N_{\alpha}$ we have $\left|A \cap N_{\alpha}\right| \leq k$ because $\mathcal{A}$ is $(k+1, \nu)$ almost disjoint. So if $A \in \mathcal{A}_{\alpha}$ then $\left|A \cap Y_{\alpha}\right|>(n+1) \cdot k-k=n \cdot k$, consequently $\mathcal{A}_{\alpha}^{\prime} \subset\left[Y_{\alpha}\right]^{<\omega} \backslash\left[Y_{\alpha}\right]^{\leq n \cdot k}$ and, clearly, $\mathcal{A}_{\alpha}^{\prime}$ is $(k+1, \nu)$-almost disjoint.

We next define, for each $\alpha<\kappa^{+n}$, a function $f_{\alpha}: Y_{\alpha} \rightarrow \kappa$, using transfinite induction as follows. Assume that $f_{\xi}$ has been defined for 
each $\xi<\alpha<\kappa^{+n}$ and set $f_{<\alpha}=\cup\left\{f_{\xi}: \xi<\alpha\right\}$. For any $A^{\prime} \in \mathcal{A}_{\alpha}^{\prime}$ let

$$
g_{\alpha}\left(A^{\prime}\right)=\bigcup\left\{f_{<\alpha}[A] \cup g(A): A \in \mathcal{A}_{\alpha} \wedge A \cap Y_{\alpha}=A^{\prime}\right\} .
$$

Since $\left|A^{\prime}\right|>n \cdot k \geq k$ (recall that $n \geq 1$ !) and $\mathcal{A}$ is $(k+1, \nu)$-almost disjoint, $\left|\left\{A \in \mathcal{A}_{\alpha}: A \cap Y_{\alpha}=A^{\prime}\right\}\right|<\nu$ and hence $g_{\alpha}\left(A^{\prime}\right) \in[\kappa]^{<\nu}$, using that $\nu$ is regular.

Thus, the inductive assumption $(*)_{n}$ can be applied to $\mathcal{A}_{\alpha}^{\prime}$ and $g_{\alpha}$ and yields us a function $f_{\alpha}: Y_{\alpha} \rightarrow \kappa$ such that

$$
I_{f_{\alpha}}\left(A^{\prime}\right) \backslash g_{\alpha}\left(A^{\prime}\right) \neq \emptyset
$$

for each $A^{\prime} \in \mathcal{A}_{\alpha}^{\prime}$.

Finally, let $f=\cup\left\{f_{\alpha}: \alpha<\kappa^{+n}\right\}$. Then for every $A \in \mathcal{A}_{\alpha}$ we have

$$
I_{f}(A) \backslash g(A) \supset I_{f_{\alpha}}\left(A \cap Y_{\alpha}\right) \backslash g_{\alpha}\left(A \cap Y_{\alpha}\right) \neq \emptyset,
$$

hence we are done because $\mathcal{A}=\bigcup\left\{\mathcal{A}_{\alpha}: \alpha<\kappa^{+n}\right\}$.

We now give a consistency result in the spirit of theorem 2.1 that uses Martin's axiom.

Theorem 2.2. Assume $M A_{\lambda}(K)$, i.e. $M A_{\lambda}$ for partial orders satisfying property $K$. Then for every natural number $k$ and for every $(k+1, \omega)$-almost disjoint system $\mathcal{A} \subset[\lambda]^{<\omega}$ such that $|A|>2 k$ for all $A \in \mathcal{A}$ we have $\chi_{\mathrm{CF}}(\mathcal{A}) \leq \omega$.

Proof. We first define the poset $\mathcal{P}_{\mathcal{A}}=\left\langle P_{\mathcal{A}}, \leq\right\rangle$ as follows: A function $f \in F n(\lambda, \omega)$ (that is a finite partial function from $\lambda$ to $\omega$ ) is in $P_{\mathcal{A}}$ iff $I_{f}(A) \neq \emptyset$ whenever $A \in \mathcal{A}$ and $A \subset \operatorname{dom}(f)$. We then let $f \leq g$ iff $f \supset g$.

We claim that the poset $\mathcal{P}_{\mathcal{A}}$ satisfies property $K$. Indeed, assume that $\left\{f_{\alpha}: \alpha<\omega_{1}\right\} \subset P_{\mathcal{A}}$. Without loss of generality we can assume that

$$
f_{\alpha}=f \cup^{*} f_{\alpha}^{\prime}, \text { and } \operatorname{dom}\left(f_{\alpha}^{\prime}\right) \cap \operatorname{dom}\left(f_{\beta}^{\prime}\right)=\emptyset \text { for }\{\alpha, \beta\} \in\left[\omega_{1}\right]^{2} .
$$

For each $\alpha<\omega_{1}$ then $\mathcal{A}_{\alpha}=\left\{A \in \mathcal{A}:\left|A \cap \operatorname{dom}\left(f_{\alpha}\right)\right|>k\right\}$ is finite because $\mathcal{A}$ is $(k+1, \omega)$-almost disjoint. Let

$$
F(\alpha)=\left\{\beta<\omega_{1}: \operatorname{dom}\left(f_{\beta}^{\prime}\right) \cap \cup \mathcal{A}_{\alpha} \neq \emptyset\right\},
$$

then $F(\alpha)$ is also finite. So by the (simplest case of the) free set theorem for set mappings we can find a set $S \in\left[\omega_{1}\right]^{\omega_{1}}$ such that $\alpha \notin F(\beta)$ and $\beta \notin F(\alpha)$ whenever $\{\alpha, \beta\} \in[S]^{2}$.

We claim that $f=f_{\alpha} \cup f_{\beta} \in P_{\mathcal{A}}$, hence $f_{\alpha}$ and $f_{\beta}$ are compatible, for any such pair $\{\alpha, \beta\}$. By (11), $f$ is a function. So assume now that $A \in \mathcal{A}$ with $A \subset \operatorname{dom}(f)$. Since $|A|>2 k$ we can assume that e.g. 
$\left|A \cap \operatorname{dom}\left(f_{\alpha}\right)\right|>k$, that is $A \in \mathcal{A}_{\alpha}$, hence $A \cap \operatorname{dom}\left(f_{\beta}^{\prime}\right)=\emptyset$. But then $A \subset \operatorname{dom}\left(f_{\alpha}\right)$ and so $I_{f}(A)=I_{f_{\alpha}}(A) \neq \emptyset$. Thus $f \in P_{\mathcal{A}}$, completing the proof that $P_{\mathcal{A}}$ has property $K$.

The rest of the proof is a standard density argument that we leave to the reader.

Remark: A slightly weaker statement than theorem [2.2, for the chromatic number $\chi$ instead of the conflict free chromatic number $\chi_{\mathrm{CF}}$, was proved in [2, Theorem 5.6]. It was asked there, in Problem 2, if the statement remains true for $\left(k, \omega_{1}\right)$-almost disjoint families. We still do not know the answer to this.

\section{LOWER BOUNDS}

We start this section with presenting a result which implies that the assumptions on the set systems formulated in theorems 2.1 and 2.2, namely that their members should be "suitably large", are really necessary.

Theorem 3.1. Assume that $\lambda \geq \omega$ and $\mu$ are cardinals, $n \geq 2, k \geq 1$ are natural numbers such that the partition relation

$$
\lambda \rightarrow(n)_{\mu^{k}}^{n-1}
$$

holds true. (Of course, if $\mu$ is infinite then $\mu^{k}=\mu$.) Then we have $\chi_{\mathrm{CF}}(\lambda, t, k+1)>\mu$ for every number $t$ satisfying $k<t \leq n \cdot k$ if $n>2$ and for every even number $t$ satisfying $k<t \leq 2 \cdot k$ if $n=2$.

Proof. Let us put $H=[\lambda]^{n-1} \times k$, then $|H|=\lambda$. We shall construct a $(k+1)$-almost disjoint family $\mathcal{A} \subset[H]^{t}$ of cardinality $\lambda$ which does not have a conflict free coloring with $\mu$ colors.

For each $Y \in[\lambda]^{n}$ we may choose a $t$-element set $A_{Y} \in\left[[Y]^{n-1} \times k\right]^{t}$ such that for every $i<k$ we have

$$
\left|\left\{B \in[Y]^{n-1}:\langle B, i\rangle \in A_{Y}\right\}\right| \neq 1 .
$$

This is easy to check and this is the point where $t$ has to be even in case $n=2$. Let us now set

$$
\mathcal{A}=\left\{A_{Y}: Y \in[\lambda]^{n}\right\} \subset[H]^{t},
$$

then clearly $|\mathcal{A}|=\lambda$.

Since $\left|[Y]^{n-1} \cap[Z]^{n-1}\right| \leq 1$ for distinct $Y, Z \in[\lambda]^{n}$, we clearly have $\left|A_{Y} \cap A_{Z}\right| \leq k$, hence $\mathcal{A}$ is $(k+1)$-almost disjoint, i.e. $\mathcal{A}$ is a $(\lambda, t, k+1)$-system. Now, it remains to show that $\chi_{\mathrm{CF}}(\mathcal{A})>\mu$.

Assume that $f: H \rightarrow \mu$ is given and define the map

$$
g:[\lambda]^{n-1} \rightarrow{ }^{k} \mu
$$


by the stipulation $g(B)(i)=f(\langle B, i\rangle)$. By our partition relation hypothesis then there is a $g$-homogeneous set $Y \in[\lambda]^{n}$. Consider an arbitrary $\langle B, i\rangle \in A_{Y}$. By (2) there is a $B^{\prime} \neq B$ with $\left\langle B^{\prime}, i\right\rangle \in A_{Y}$ as well, hence we have $f(\langle B, i\rangle)=g(B)(i)=g\left(B^{\prime}\right)(i)=f\left(\left\langle B^{\prime}, i\right\rangle\right)$. Since $\langle B, i\rangle$ was arbitrary we obtain that $f$ is not a conflict free coloring of $\mathcal{A}$, completing the proof.

We now list a number of easy but quite useful corollaries of theorem 3.1 .

Corollary 3.2. If $\lambda=\omega$ or $\lambda$ is weakly compact then for any $2 \leq d \leq$ $t<\omega$ we have $\chi_{\mathrm{CF}}(\lambda, t, d)=\lambda$.

Proof. To see this, let us first choose a natural number $n>2$ such that $t \leq n \cdot(d-1)$. By our choice of $\lambda$, for every $\mu<\lambda$ we have $\lambda \rightarrow(n)_{\mu^{d-1}}^{n-1}$, in fact even $\lambda \rightarrow(\lambda)_{\mu^{d-1}}^{n-1}$. But then theorem 3.1 immediately yields $\chi_{\mathrm{CF}}(\lambda, t, d)>\mu$, hence as $\mu<\lambda$ was arbitrary, $\chi_{\mathrm{CF}}(\lambda, t, d)=\lambda$.

Since $\chi_{\mathrm{CF}}\left(\omega_{1}, t, 2\right) \geq \chi_{\mathrm{CF}}(\omega, t, 2)$, it immediately follows from 3.2 and the case $n=2, k=1$ of theorem 2.1 that $\chi_{\mathrm{CF}}\left(\omega_{1}, t, 2\right)=\omega$ whenever $3 \leq t<\omega$. Similarly, comparing theorem 2.2 with corollary 3.2 we may conclude that $M A_{\lambda}(K)$ implies $\chi_{\mathrm{CF}}(\lambda, t, d)=\omega$ whenever $d \geq 2$ and $t>2(d-1)$.

An analogous argument as in the proof of corollary [3.2, using the case $n=2$ of theorem 3.1 and the trivial partition relation $\lambda \rightarrow(2)_{\kappa}^{1}$ for all $\kappa<\lambda$, yields the following result.

Corollary 3.3. If $\lambda$ is infinite and $1 \leq k<\omega$, then

$$
\chi_{\mathrm{CF}}(\lambda, 2 k, k+1)=\lambda .
$$

On the basis of the conjecture that $\chi_{\mathrm{CF}}(\lambda, \kappa, \mu)$ is monotone decreasing in its second argument, it is natural to expect from 3.3 that we also have $\chi_{\mathrm{CF}}(\lambda, 2 k-1, k+1)=\lambda$. We shall show below that this is indeed true for "most" values of $\lambda$, however the full statement remains open in ZFC. We first give a somewhat technical lemma.

Lemma 3.4. Let $\lambda$ be a cardinal that admits a coloring $f:[\lambda]^{2} \rightarrow \lambda$ of its pairs such that for any partition $\mathcal{P}$ of $\lambda$ with $|\mathcal{P}|<\lambda$ there are $P \in \mathcal{P}$ and $\{\alpha, \beta, \gamma\} \in[P]^{3}$ satisfying $f\{\alpha, \beta\}=\gamma$. Then, for any $k>1$, we have

$$
\chi_{\mathrm{CF}}(\lambda, 2 k-1, k+1)=\lambda .
$$

Proof.

$$
\mathcal{I}(f)=\left\{\{\alpha, \beta\} \in[\lambda]^{2}: f\{\alpha, \beta\} \notin\{\alpha, \beta\}\right\}
$$


naturally decomposes into the following three parts:

$$
\begin{aligned}
& \mathcal{I}_{0}(f)=\left\{\{\alpha, \beta\} \in[\lambda]^{2}: f\{\alpha, \beta\}<\alpha<\beta\right\}, \\
& \mathcal{I}_{1}(f)=\left\{\{\alpha, \beta\} \in[\lambda]^{2}: \alpha<f\{\alpha, \beta\}<\beta\right\}, \\
& \mathcal{I}_{2}(f)=\left\{\{\alpha, \beta\} \in[\lambda]^{2}: \alpha<\beta<f\{\alpha, \beta\}\right\} .
\end{aligned}
$$

We claim that our assumption on $f$ may be strengthened as follows: There is a fixed $j<3$ such that for any partition $\mathcal{P}$ of $\lambda$ with $|\mathcal{P}|<\lambda$ there are $P \in \mathcal{P}$ and $\{\alpha, \beta\} \in \mathcal{I}_{j}(f) \cap[P]^{2}$ for which $f\{\alpha, \beta\} \in P$.

Indeed, for every $j<3$ let $g_{j}:[\lambda]^{2} \rightarrow \lambda$ be chosen in such a way that $g_{j}$ extends $f \uparrow \mathcal{I}_{j}(f)$. Then for one $j<3$ the coloring $g_{j}$ together with its index $j$ must satisfy the claim. Otherwise for every $j<3$ there is a partition $\mathcal{P}_{j}$ of $\lambda$ with $\left|\mathcal{P}_{j}\right|<\lambda$ such that $g_{j}\{\alpha, \beta\} \notin P$ whenever $\{\alpha, \beta\} \in \mathcal{I}_{j}\left(g_{j}\right) \cap[P]^{2}$. But then

$$
\mathcal{P}=\left\{P_{1} \cap P_{2} \cap P_{3}: P_{j} \in \mathcal{P}_{j}, j<3\right\}
$$

is a partition of $\lambda$ with $|\mathcal{P}|<\lambda$ that cannot satisfy our original assumption on $f$, a contradiction. So from here on we assume that $f$ has the stronger property with $j$ fixed.

Take $\lambda$ many pairwise disjoint sets of size $k-1,\left\{H_{\alpha}: \alpha<\lambda\right\}$, and for each $\alpha<\lambda$ fix a member $h_{\alpha} \in H_{\alpha}$. For each $\{\alpha, \beta\} \in \mathcal{I}(f)$ let

$$
A_{\{\alpha, \beta\}}=H_{\alpha} \cup H_{\beta} \cup\left\{h_{f\{\alpha, \beta\}}\right\} .
$$

It is easy to check that then $\mathcal{A}=\left\{A_{\{\alpha, \beta\}}:\{\alpha, \beta\} \in \mathcal{I}_{j}(f)\right\}$ is a $(\lambda, 2 k-1, k+1)$-system and we claim that $\chi_{\mathrm{CF}}(\mathcal{A})=\lambda$.

Indeed, consider any map $g: \cup \mathcal{A} \rightarrow \kappa$ with $\kappa<\lambda$. Then, by our assumption, there is a pair $\{\alpha, \beta\} \in \mathcal{I}_{j}(f)$ such that

$$
g\left[H_{\alpha}\right]=g\left[H_{\beta}\right]=g\left[H_{f\{\alpha, \beta\}} .\right.
$$

But clearly, every value taken by $g$ on $A_{\{\alpha, \beta\}}$ is taken at least twice, consequently $g$ is not a conflict free coloring of $\mathcal{A}$.

Let us note that if $\lambda$ is regular and $f:[\lambda]^{2} \rightarrow \lambda$ establishes the negative partition relation

$$
\lambda \nrightarrow[\lambda]_{\lambda}^{2}
$$

that is, $f[X]=\lambda$ for every $X \in[\lambda]^{\lambda}$, then $f$ trivially satisfies the requirement of lemma 3.4 as well. Moreover, it is known that $\lambda \nrightarrow[\lambda]_{\lambda}^{2}$ is valid whenever $\lambda=\kappa^{+}$for a regular cardinal $\kappa$, see e.g. [14]. Thus, we immediately obtain the following result.

Corollary 3.5. If $\lambda$ is either a limit cardinal or the successor of a regular cardinal and $1<k<\omega$ then

$$
\chi_{\mathrm{CF}}(\lambda, 2 k-1, k+1)=\lambda .
$$


The following corollary of theorem 3.1 uses, for $r=n-2>0$, the well-known Erdős-Rado partition theorem

$$
\beth_{r}(\kappa)^{+} \rightarrow\left(\kappa^{+}\right)_{\kappa}^{r+1} .
$$

Recall that $\beth_{r}(\kappa)$ is defined by the recursion $\beth_{0}(\kappa)=\kappa, \beth_{r+1}(\kappa)=$ $2^{\beth_{r}(\kappa)}$.

Corollary 3.6. If $n \geq 3$ and $k<t \leq n \cdot k$ then, for every $\kappa \geq \omega$,

$$
\chi_{\mathrm{CF}}\left(\beth_{n-2}(\kappa)^{+}, t, k+1\right)>\kappa .
$$

Consequently, if $\lambda$ is strong limit then for any $2 \leq d \leq t<\omega$ we have $\chi_{\mathrm{CF}}(\lambda, t, d)=\lambda$.

Proof. The first part, as mentioned, follows immediately from theorem 3.1 and the Erdős-Rado partition theorem. To see the second, consider any $\kappa<\lambda$ and choose $n \geq 3$ such that $t \leq n \cdot(d-1)$. Then, by the first part, we have $\chi_{\mathrm{CF}}\left(\beth_{n-2}(\kappa)^{+}, t, k+1\right)>\kappa$, moreover $\beth_{n-2}(\kappa)^{+}<\lambda$ as $\lambda$ is strong limit, hence $\chi_{\mathrm{CF}}(\lambda, t, d)>\kappa$ as well. This completes the proof as $\kappa<\lambda$ was arbitrary.

Our next result yields a lower bound for $\chi_{\mathrm{CF}}(\lambda, t, k+1)$ for $t \leq 2 k$, like corollaries 3.3 and 3.5 . Of course, if the statement of corollary 3.5 turns out to be valid for all $\lambda$, as we expect, then it becomes superfluous.

Theorem 3.7. Assume that $\lambda$ and $\mu$ are infinite cardinals such that $\lambda^{<\mu}=\lambda$, moreover $0<k<t \leq 2 k$ are natural numbers. Then

$$
\chi_{\mathrm{CF}}(\lambda, t, k+1) \geq \mu \text {. }
$$

Proof. We are going to construct a $(\lambda, t, k+1)$-system $\mathcal{A} \subset[\lambda]^{t}$ that satisfies the following property $\Phi(\lambda, \mu, k, t)$ :

For every $Y \in[\lambda]^{<\mu}$ and for every disjoint collection $B \subset[\lambda]^{k}$ with $|B|<\mu$ there is a set $x \in[\lambda \backslash Y]^{t-k}$ such that $x \cup b \in \mathcal{A}$ for each $b \in B$.

Before doing this, however, let us show that if $\mathcal{A}$ satisfies $\Phi(\lambda, \mu, k, t)$ then $\chi_{\mathrm{CF}}(\mathcal{A}) \geq \mu$. Indeed, let $f: \lambda \rightarrow \nu$ be given for some $\nu<\mu$, where $\nu$ is infinite if $\mu>\omega$. Let us put $S=\left\{\zeta<\nu:\left|f^{-1}\{\zeta\}\right| \geq \omega\right\}$ if $\mu=\omega$ and $S=\left\{\zeta<\nu:\left|f^{-1}\{\zeta\}\right| \geq \nu\right\}$ otherwise. We also set $Y=\bigcup\left\{f^{-1}\{\zeta\}: \zeta \in \nu \backslash S\right\}$, clearly then $|Y|<\mu$. Next we consider the collection $\mathcal{S}=\{z \subset S: 0<|z| \leq t-k\}$, again we have $|\mathcal{S}|<\mu$. It is straight-forward to check that we may select for each $z \in \mathcal{S}$ a set $b_{z} \in[\lambda \backslash Y]^{k}$ so that $f\left[b_{z}\right]=z$, moreover $B=\left\{b_{z}: z \in \mathcal{S}\right\}$ is disjoint.

By $\Phi(\lambda, \mu, k, t)$ there is some $x \in[\lambda \backslash Y]^{t-k}$ such that $x \cup b_{z} \in \mathcal{A}$ for each $z \in \mathcal{S}$. Now, $x \cap Y=\emptyset$ implies that $z=f[x] \in \mathcal{S}$, hence $x \cup b_{z} \in \mathcal{A}$. But, as $x \cap b_{z}=\emptyset$, the equality $f[x]=f\left[b_{z}\right](=z)$ witnesses that $f$ is not a conflict free coloring of $\mathcal{A}$, hence $\chi_{\mathrm{CF}}(\mathcal{A}) \geq \mu$. 
Now, we show how to construct $\mathcal{A}$ satisfying $\Phi(\lambda, \mu, k, t)$ by a transfinite recursion of length $\lambda$. To start with, we fix a $\lambda$-type enumeration of $[\lambda]^{<\mu} \times \mathcal{B}$ :

$$
[\lambda]^{<\mu} \times \mathcal{B}=\left\{\left\langle Y_{\alpha}, B_{\alpha}\right\rangle: \alpha<\lambda\right\},
$$

where $\mathcal{B}$ is the family of all disjoint collections $B \subset[\lambda]^{k}$ with $|B|<\mu$. This is possible because $\lambda^{<\mu}=\lambda$.

Next, assume that $\alpha<\lambda$ and for each $\beta<\alpha$ we have already constructed a $(k+1)$-almost disjoint family $\mathcal{A}_{\beta} \subset[\lambda]^{t}$ such that $\left|\mathcal{A}_{\beta}\right| \leq$ $\mu \cdot|\beta|$ if $\mu<\lambda$ and $\left|\mathcal{A}_{\beta}\right|<\lambda$ if $\mu=\lambda$. We also assume that $\mathcal{A}_{\beta} \subset \mathcal{A}_{\gamma}$ whenever $\beta<\gamma<\alpha$.

Now, if $\alpha$ is limit then we simply put $\mathcal{A}_{\alpha}=\cup_{\beta<\alpha} \mathcal{A}_{\beta}$. It is easy to see that then all our inductive hypotheses remain valid. This is obvious if $\mu<\lambda$, and if $\mu=\lambda$ then it follows because $\lambda$ is regular by the assumption $\lambda^{<\lambda}=\lambda$.

If, on the other hand, $\alpha=\beta+1$ then we consider the pair $\left\langle Y_{\beta}, B_{\beta}\right\rangle$ and choose a set $x \in[\lambda]^{t-k}$ that is disjoint from $\cup \mathcal{A}_{\beta} \cup \cup B_{\beta} \cup Y_{\beta}$. Then we put

$$
\mathcal{A}_{\alpha}=\mathcal{A}_{\beta+1}=\mathcal{A}_{\beta} \cup\left\{b \cup x: b \in B_{\beta}\right\} .
$$

Again, it is obvious that our inductive hypotheses remain valid.

Finally, if the transfinite recursion is completed, then we set

$$
\mathcal{A}=\bigcup\left\{\mathcal{A}_{\alpha}: \alpha<\lambda\right\} .
$$

It is obvious from our construction that $\mathcal{A} \subset[\lambda]^{t}$ is a $(\lambda, t, k+1)$-system that satisfies property $\Phi(\lambda, \mu, k, t)$ and hence $\chi_{\mathrm{CF}}(\mathcal{A}) \geq \mu$.

Corollary 3.8. Let $k$ and $t$ be integers with $1 \leq k<t \leq 2 k$. If $\kappa^{+}=2^{\kappa}$ then $\chi_{\mathrm{CF}}\left(\kappa^{+}, t, k+1\right)=\kappa^{+}$.

In particular, as we promised, $\mathrm{CH}$ implies $\chi_{\mathrm{CF}}\left(\omega_{1}, t, k+1\right)=\omega_{1}$ for any such $k$ and $t$. Actually, our previous results enable us to give, under the assumption of $\mathrm{GCH}$, a complete and rather attractive description of the behavior of $\chi_{\mathrm{CF}}(\lambda, t, k+1)$ for all $\lambda \geq \omega>t>k \geq 1$.

Theorem 3.9. Assume $G C H$ and let $\kappa$ be any limit cardinal or $\kappa=\omega$, moreover fix the natural number $k \geq 1$. Then for any $n<\omega$ we have

$$
\chi_{\mathrm{CF}}\left(\kappa^{+n}, t, k+1\right)=\left\{\begin{array}{rr}
\kappa^{+(n+1-i)} & \text { if } i \cdot k<t \leq(i+1) \cdot k, \\
& i=1, \ldots, n ; \\
\kappa & \text { if }(n+1) \cdot k<t .
\end{array}\right.
$$


Proof. Let us note first that by the second part of corollary 3.6 and by corollary 3.2 we have $\chi_{\mathrm{CF}}(\kappa, t, k+1)=\kappa$ for all $0<k<t<\omega$ which shows that our claim holds for $n=0$. So, from here on we fix $n \geq 1$.

Let us assume now that $k<t \leq 2 k$. In this case we may apply corollary 3.8 to $\kappa^{+n}=2^{\left(\kappa^{+n-1}\right)}$ and conclude that

$$
\chi_{\mathrm{CF}}\left(\kappa^{+n}, t, k+1\right)=\kappa^{+n}=\kappa^{+(n+1-1)} .
$$

Next, consider the case $i \cdot k<t \leq(i+1) \cdot k$ with $2 \leq i \leq n$. Then from $i \cdot k<t$, applying theorem 2.1 to the cardinal $\kappa^{+(n+1-i)}$ and the number $i$, we obtain $\chi_{\mathrm{CF}}\left(\kappa^{+n}, t, k+1\right) \leq \kappa^{+(n+1-i)}$. ¿From $t \leq(i+1) \cdot k$, on the other hand, applying corollary 3.6 to the number $i+1 \geq 3$ and the cardinal $\kappa^{+(n-i)}$ we obtain the converse inequality $\chi_{\mathrm{CF}}\left(\kappa^{+n}, t, k+1\right) \geq \kappa^{+(n+1-i)}$.

Finally, assume that $t>(n+1) \cdot k$. Then from theorem 2.1, applied with the number $n+1$, we conclude $\chi_{\mathrm{CF}}\left(\kappa^{+n}, t, k+1\right) \leq \kappa$. But then we must have $\chi_{\mathrm{CF}}\left(\kappa^{+n}, t, k+1\right)=\kappa$ because already $\chi_{\mathrm{CF}}(\kappa, t, k+1)=\kappa$.

This concludes the proof because we have checked all the cases.

It is immediate from theorem 3.9 that, in accordance with our earlier conjecture, $\chi_{\mathrm{CF}}(\lambda, t, d)$ is a monotone decreasing function of $t<\omega$ for fixed $\lambda$ and $d$, at least if GCH holds.

Problem 3.1. Is $\chi_{\mathrm{CF}}(\lambda, 2 k-1, k+1)=\lambda$ provable in $Z F C$ for all $\lambda \geq \omega$ and $1<k<\omega$ ?

\section{PART II. The CASE $\lambda \geq \kappa \geq \omega>\mu$}

\section{4. $\omega$ COLORS SUFFICE}

It follows from theorem 2.1 that if $\lambda<\aleph_{\omega}$ then, for fixed $d<\omega$, we have $\chi_{\mathrm{CF}}(\lambda, t, d) \leq \omega$ provided that $t<\omega$ is large enough. The result we prove in this section shows that if we replace $t$ with any infinite cardinal $\kappa$ then $\chi_{\mathrm{CF}}(\lambda, \kappa, d) \leq \omega$ holds for all $\lambda \geq \kappa$.

Theorem 4.1. For any $\lambda \geq \kappa \geq \omega$ and $d<\omega$ we have $\chi_{\mathrm{CF}}(\lambda, \kappa, d) \leq$ $\omega$, in fact even the stronger relation $[\lambda, \kappa, d] \Rightarrow \omega$.

First proof of Theorem 4 .1. We prove $[\lambda, \kappa, d] \Rightarrow \omega$ by transfinite induction on $\kappa$ and $\lambda$ : Assuming $\left[\kappa^{\prime}, \kappa^{\prime}, d\right] \Rightarrow \omega$ and $\left[\lambda^{\prime}, \kappa, d\right] \Rightarrow \omega$ for all $\omega \leq \kappa^{\prime}<\kappa$ and $\kappa \leq \lambda^{\prime}<\lambda$, we deduce $[\lambda, \kappa, d] \Rightarrow \omega$.

Case 1: $\lambda=\kappa=\omega$.

Let $\mathcal{A}=\left\{A_{n}: n<\omega\right\} \subset[\omega]^{\omega}$ be $d$-almost disjoint (actually, $\omega$-almost 
disjoint would suffice) and construct $c: \omega \rightarrow \omega$ in such a way that $c \nmid A_{n} \backslash \cup\left\{A_{m}: m<n\right\}$ is a bijection with range $\omega$ for each $n<\omega$. Thus $\omega \backslash I_{c}\left(A_{n}\right) \subset c\left[A_{n} \cap \cup\left\{A_{m}: m<n\right\}\right]$ is finite for all $A_{n} \in \mathcal{A}$, and we are done.

Case 2: $\lambda=\kappa>\omega$.

Let $\mathcal{A} \subset[\kappa]^{\kappa}$ be $d$-almost disjoint and $\left\langle N_{\alpha}: \alpha<\kappa\right\rangle$ be a $\kappa$-chain of elementary submodels with $\mathcal{A} \in N_{1}$. For $\alpha<\kappa$ let $\kappa_{\alpha}=\left|N_{\alpha+1}\right|$, $B_{\alpha}=\cup\left(\mathcal{A} \cap N_{\alpha}\right)$ and $Y_{\alpha}=N_{\alpha+1} \cap\left(\kappa \backslash\left(B_{\alpha} \cup N_{\alpha}\right)\right)$.

If $A \in \mathcal{A} \cap N_{\alpha+1} \backslash N_{\alpha}$ then

$$
\left|A \cap B_{\alpha}\right| \leq \sum\left\{\left|A \cap A^{\prime}\right|: A^{\prime} \in \mathcal{A} \cap N_{\alpha}\right\} \leq\left|N_{\alpha}\right| \cdot d<\kappa
$$

and so $\left|\kappa \backslash\left(B_{\alpha} \cup N_{\alpha}\right)\right|=\left|A \backslash\left(B_{\alpha} \cup N_{\alpha}\right)\right|=\kappa$. But $A \backslash\left(B_{\alpha} \cup N_{\alpha}\right) \in N_{\alpha+1}$ and $\kappa_{\alpha} \subset N_{\alpha+1}$ imply

$$
\left|Y_{\alpha}\right|=\left|A \cap Y_{\alpha}\right|=\left|N_{\alpha+1} \cap\left(A \backslash\left(B_{\alpha} \cup N_{\alpha}\right)\right)\right|=\kappa_{\alpha},
$$

consequently

$$
\mathcal{A}_{\alpha}=\left\{A \cap Y_{\alpha}: A \in \mathcal{A} \cap N_{\alpha+1} \backslash N_{\alpha}\right\} \subset\left[Y_{\alpha}\right]^{\kappa_{\alpha}},
$$

and $\mathcal{A}_{\alpha}$ is clearly $d$-almost disjoint. By the inductive assumption $\left[\kappa_{\alpha}, \kappa_{\alpha}, d\right] \Rightarrow \omega$, there is a function $c_{\alpha}: Y_{\alpha} \rightarrow \omega$ such that $\omega \backslash I_{c_{\alpha}}\left(A^{\prime}\right)$ is finite for all $A^{\prime} \in \mathcal{A}_{\alpha}$.

Let $c^{\prime}=\cup\left\{c_{\alpha}: \alpha<\kappa\right\}$ and consider the function $c \supset c^{\prime}$ which maps $\lambda$ into $\omega$ in such a way that $c\left[\lambda \backslash \operatorname{dom}\left(c^{\prime}\right)\right] \subset\{0\}$. Now, let $A \in \mathcal{A}$ and $\alpha<\kappa$ be such that $A \in N_{\alpha+1} \backslash N_{\alpha}$. Then $A^{\prime}=A \cap Y_{\alpha} \in \mathcal{A}_{\alpha}$, so $\omega \backslash I_{c_{\alpha}}\left(A^{\prime}\right)$ is finite. But we also have

$$
\left|A \cap \operatorname{dom}\left(c^{\prime} \backslash c_{\alpha}\right)\right|<d .
$$

Indeed, if $\alpha<\beta<\kappa$ then $A \cap Y_{\beta}=\emptyset$, while $A \notin N_{\alpha}$ implies $\left|A \cap N_{\alpha}\right|<$ $d$, and hence $\left|A \cap \cup\left\{Y_{\gamma}: \gamma<\alpha\right\}\right|<d$ as well. Since

$$
I_{c_{\alpha}}\left(A^{\prime}\right) \backslash I_{c}(A) \subset c^{\prime}\left[A \backslash \operatorname{dom} c_{\alpha}\right] \cup\{0\},
$$

it follows that $\omega \backslash I_{c}(A)$ is finite, and we are done.

Case 3: $\lambda>\kappa$.

Let $\mathcal{A} \subset[\lambda]^{\kappa}$ be $d$-almost disjoint and $\left\langle N_{\alpha}: \alpha<\lambda\right\rangle$ be a $\lambda$-chain of elementary submodels with $(\kappa+1) \cup\{\mathcal{A}\} \subset N_{1}$. For each $\alpha<\lambda$ let $Y_{\alpha}=\lambda \cap N_{\alpha+1} \backslash N_{\alpha}$, then $\kappa \leq\left|Y_{\alpha}\right|=\left|N_{\alpha+1}\right|<\lambda$.

For any $A \in \mathcal{A} \cap N_{\alpha+1} \backslash N_{\alpha}$ we have $\left|A \cap N_{\alpha}\right|<d$ and $A \subset N_{\alpha+1}$, hence

$$
\mathcal{A}_{\alpha}=\left\{A \backslash N_{\alpha}: A \in \mathcal{A} \cap N_{\alpha+1} \backslash N_{\alpha}\right\} \subset\left[Y_{\alpha}\right]^{\kappa},
$$

and $\mathcal{A}_{\alpha}$ is $d$-almost disjoint. Now, we may argue inductively, exactly as in Case 2, to obtain a map $c: \lambda \rightarrow \omega$ such that $\omega \backslash I_{c}(A)$ is finite for each $A \in \mathcal{A}$. 
Remark . P. Komjáth pointed out to us an easy proof of Theorem 4.1 for the case $\kappa=\omega$. His proof relied on a result of his proved in 10] claiming that every $(\lambda, \omega, d)$-system $\mathcal{A}$ is essentially disjoint, i.e. one can omit a finite set $F(A)$ from each element $A$ of $\mathcal{A}$ in such a way that the sets $A \backslash F(A)$ are pairwise disjoint. By taking a bijection between $A \backslash F(A)$ and $\omega$ for each $A \in \mathcal{A}$, and then coloring the rest arbitrarily, we get an appropriate $\omega$-coloring. Based on this observation, and a result of Erdős and Hajnal, we shall give a short alternative proof of theorem 4.1 .

We recall from [7] and [8] that a set $X$ is called a $\tau$-transversal of a family $\mathcal{A}$ if $0<|X \cap A|<\tau$ for all $A \in \mathcal{A}$. Moreover, the symbol $\mathbf{M}(\lambda, \kappa, \mu) \rightarrow \mathbf{B}(\tau)$ is used there to denote the statement that every $(\lambda, \kappa, \mu)$-system has a $\tau$-transversal.

For us it will be useful to introduce the following variation on this concept: We say that $X$ is a $\tau$-witness for $\mathcal{A}$ iff $|X \cap A|=\tau$ for all $A \in \mathcal{A}$. Clearly, any $\tau$-witness is a $\tau^{+}$-transversal. It is easy to see that if $\kappa \geq \tau \geq \omega$ then $\mathbf{M}(\lambda, \kappa, \mu) \rightarrow \mathbf{B}\left(\tau^{+}\right)$holds iff every $(\lambda, \kappa, \mu)$-system has a $\tau$-witness.

Definition 4.2. A $(\lambda, \kappa)$-family $\mathcal{A}$ is called essentially disjoint (ED, in short) iff for each $A \in \mathcal{A}$ there is a set $F(A) \in[A]^{<\kappa}$ such that the family $\{A \backslash F(A): A \in \mathcal{A}\}$ is disjoint. $\mathbf{M}(\lambda, \kappa, \mu) \rightarrow \mathbf{E D}$ denotes the statement that every $(\lambda, \kappa, \mu)$-system is $\mathrm{ED}$.

Proposition 4.3. Assume $\mu \leq \tau \leq \kappa \leq \lambda$ and $\tau \geq \omega$. Then

$$
\mathbf{M}(\lambda, \kappa, \mu) \rightarrow \mathbf{B}\left(\tau^{+}\right) \text {and } \mathbf{M}(\lambda, \tau, \mu) \rightarrow \mathbf{E D}
$$

together imply $[\lambda, \kappa, \mu] \Rightarrow \tau$.

Proof. Let $\mathcal{A} \subset[\lambda]^{\kappa}$ be a $(\lambda, \kappa, \mu)$-system. Since $\mathbf{M}(\lambda, \kappa, \mu) \rightarrow \mathbf{B}\left(\tau^{+}\right)$ there is a $\tau$-witness $X$ for $\mathcal{A}$. Then

$$
\mathcal{A}^{\prime}=\mathcal{A} \uparrow X=\{A \cap X: A \in \mathcal{A}\} \subset[\lambda]^{\tau}
$$

is a $(\lambda, \tau, \mu)$-system. Applying $\mathbf{M}(\lambda, \tau, \mu) \rightarrow \mathbf{E D}$ for $\mathcal{A}^{\prime}$ there is a function $F: \mathcal{A}^{\prime} \rightarrow[\lambda]^{<\tau}$ such that the family $\left\{A^{\prime} \backslash F\left(A^{\prime}\right): A^{\prime} \in \mathcal{A}^{\prime}\right\}$ is disjoint.

Let $c: \lambda \rightarrow \tau$ be a function such that

(i) $c[\lambda \backslash X]=\{0\}$,

(ii) $c \uparrow A^{\prime} \backslash F\left(A^{\prime}\right)$ is a bijection between $A^{\prime} \backslash F\left(A^{\prime}\right)$ and $\tau$ for each $A^{\prime} \in \mathcal{A}^{\prime}$.

Then for each $A \in \mathcal{A}$,

$$
\mu \backslash I_{c}(A) \subset\{0\} \cup c[F(A \cap X)] \in[\tau]^{<\tau},
$$


i.e. $c$ witnesses $[\lambda, \kappa, \rho] \Rightarrow \mu$

Second proof of theorem 4.1. In [3, Theorem 8(b)] Erdös and Hajnal proved that

$$
\mathbf{M}(\lambda, \kappa, d) \rightarrow \mathbf{B}(\omega) \text { for } d<\omega \leq \kappa \leq \lambda .
$$

Moreover, in [10, Theorem 2], Komjáth proved

$$
\mathbf{M}(\lambda, \omega, d) \rightarrow \mathbf{E D} \text { for } d<\omega \leq \lambda,
$$

By proposition 4.3, (5) and (6) imply $[\lambda, \kappa, d] \Rightarrow \omega$. (Actually, instead of (5), $\mathbf{M}(\lambda, \kappa, d) \rightarrow \mathbf{B}\left(\omega_{1}\right)$ would be enough.)

As a matter of fact, the theorem of Erdös and Hajnal, 3, Theorem $8(\mathrm{~b})$ ] that we stated and used above can be proved with the method of elementary chains as presented in the first proof of theorem 4.1. Moreover, we should point out that all the results mentioned in this section can also be deduced from the very general, and therefore rather technical, main theorem 1.6 of [7].

\section{A FINITE UPPER BOUND FOR $w \chi_{\mathrm{CF}}\left(\kappa^{+m}, \kappa, d\right)$}

We have seen in the previous section that $\chi_{\mathrm{CF}}(\lambda, \kappa, d)$ is countable whenever $\lambda \geq \kappa \geq \omega>d$. The aim of this section is to show that if $\lambda$ is "not much bigger than" $\kappa$, namely it is a finite successor of $\kappa$, then $\chi_{\mathrm{CF}}(\lambda, \kappa, d)$ is even finite. This is immediate from the following theorem that is formulated in terms of the weak conflict free chromatic number.

Theorem 5.1. If $\kappa$ is infinite, $d>0$ and $m$ are natural numbers then

$$
w \chi_{\mathrm{CF}}\left(\kappa^{+m}, \kappa, d\right) \leq\left\lfloor\frac{(m+1)(d-1)+1}{2}\right\rfloor+1,
$$

or with our alternative arrow notation:

$$
\left[\kappa^{+m}, \kappa, d\right] \rightarrow_{w}\left\lfloor\frac{(m+1)(d-1)+1}{2}\right\rfloor+1 .
$$

We shall actually prove a stronger result than theorem 5.1, This involves a refined version of our weak arrow relation whose definition is given next. In this we shall use $\mathcal{F}(A, B)$ to denote the set of all partial functions from $A$ to $B$. 
Definition 5.2. Let $\lambda \geq \kappa \geq \omega$ and $d, k, x \in \omega$. Then

$$
[\lambda, \kappa, d, k] \rightarrow_{w} x
$$

abbreviates the following statement: If $C \subset \lambda$ and $\mathcal{A} \subset[\lambda]^{\kappa}$ is any $d$-almost disjoint system satisfying $|A \cap C| \leq k$ for each $A \in \mathcal{A}$, then for every partial function $f \in \mathcal{F}(C, x)$ there is a weak conflict free coloring $g \in \mathcal{F}(\lambda, x)$ of $\mathcal{A}$ such that $g\lceil C=f$. Note that the last equality is equivalent to $g \supset f$ and $C \cap \operatorname{dom}(g)=\operatorname{dom}(f)$.

For later use we also define the (strict) relation $[\lambda, \kappa, d, k] \rightarrow x$ as follows: For any $d$-almost disjoint $\mathcal{A} \subset[\lambda]^{\kappa}$ and $f \in \mathcal{F}(\lambda, x)$ satisfying $|A \cap \operatorname{dom}(f)| \leq k$ for each $A \in \mathcal{A}$, there is a conflict free coloring $g: \lambda \rightarrow x$ of $\mathcal{A}$ with $g \supset f$.

The main result of this section may be then formulated as follows. (Note that theorem 5.1 is an immediate corollary of the particular case $k=0$ of theorem [5.3.)

Theorem 5.3. Let $\kappa$ be an infinite cardinal and $m, d, k$ be natural numbers with $d>0$. Then

$$
\left[\kappa^{+m}, \kappa, d, k\right] \rightarrow_{w}\left\lfloor\frac{(m+1)(d-1)+k+1}{2}\right\rfloor+1 .
$$

The proof of theorem 5.3 will be carried out by induction on $m$, using theorems 5.4 and 5.5 below.

Theorem 5.4. Let $\kappa$ be an infinite cardinal, moreover $d$ and $x$ be natural numbers with $2 x>d$. Then

$$
[\kappa, \kappa, d, 2 x-d-1] \rightarrow_{w} x .
$$

Proof of Theorem 5.4. Let us write $k=2 x-d-1$ and assume that a set $C \subset \kappa$, a $d$-almost disjoint system $\mathcal{A} \subset[\kappa]^{\kappa}$, and a partial function $f \in \mathcal{F}(C, x)$ are given such that $|A \cap C| \leq k$ for each $A \in \mathcal{A}$. We may clearly assume that $|\mathcal{A}|=\kappa$, and hence may fix a one-to-one $\kappa$-type enumeration $\left\{A_{\eta}: \eta<\kappa\right\}$ of $\mathcal{A}$.

By transfinite induction we shall define $f_{\eta} \in \mathcal{F}(\kappa, x)$ for $\eta \leq \kappa$ such that the inductive conditions (i) - (iv) below be valid.

(i) $f_{\eta} \supset f_{\zeta} \supset f$ for $\eta>\zeta$,

(ii) $C \cap \operatorname{dom}\left(f_{\eta} \backslash f\right)=\emptyset$ and $\left|f_{\eta} \backslash f\right| \leq|\eta|$,

(iii) $\forall \zeta<\eta \exists i<x\left|A_{\zeta} \cap f_{\eta}^{-1}\{i\}\right|=1$,

(iv) if $\gamma \geq \eta$ then $\left|A_{\gamma} \cap \operatorname{dom}\left(f_{\eta} \backslash f\right)\right| \leq d$.

Case 1. $\eta=0$.

Put $f_{0}=f$, then (i) - (iv) hold trivially.

Case 2. $\eta$ is a limit ordinal.

Put $f_{\eta}=\cup\left\{f_{\zeta}: \zeta<\eta\right\}$. It is again easy to check that the validity of 
conditions (i) - (iv) will be preserved. In particular, (iii) is preserved because, as $x$ is finite, for each $\zeta<\eta$ there are cofinally many $\xi<\eta$ satisfying $\left|A_{\zeta} \cap f_{\xi}^{-1}\{i\}\right|=1$ with the same $i<x$.

Case 3. $\eta=\zeta+1$.

Then we have

$$
\left|A_{\zeta} \cap \operatorname{dom}\left(f_{\zeta}\right)\right|=\left|A_{\zeta} \cap \operatorname{dom}(f)\right|+\left|A_{\zeta} \cap\left(\operatorname{dom}\left(f_{\zeta} \backslash f\right)\right)\right| \leq k+d,
$$

consequently, $2 x>2 x-1=k+d$ implies that there is $i<x$ such that $\left|A_{\zeta} \cap f_{\zeta}^{-1}\{i\}\right| \leq 1$. If there is an $i<x$ such that $\left|A_{\zeta} \cap f_{\zeta}^{-1}\{i\}\right|=1$ then the choice $f_{\eta}=f_{\zeta}$ clearly works.

Otherwise we may fix $j<x$ with $A_{\zeta} \cap f_{\zeta}^{-1}\{j\}=\emptyset$. Let us then put

$$
\mathcal{A}_{\zeta}=\left\{A_{\xi}: \xi<\zeta\right\} \cup\left\{A_{\gamma}: \zeta<\gamma \wedge\left|A_{\gamma} \cap \operatorname{dom}\left(f_{\zeta} \backslash f\right)\right|=d\right\} .
$$

Using $\left|\operatorname{dom}\left(f_{\zeta} \backslash f\right)\right| \leq|\zeta|<\kappa$ and that $\mathcal{A}$ is $d$-almost disjoint we get $\left|\mathcal{A}_{\zeta}\right|<\kappa$, moreover we also have $\left|C \cap A_{\zeta}\right| \leq k$. Thus we can pick

$$
\xi_{\zeta} \in A_{\zeta} \backslash\left(\cup \mathcal{A}_{\zeta} \cup C\right)
$$

and put

$$
f_{\eta}=f_{\zeta+1}=f_{\zeta} \cup\left\{\left\langle\xi_{\zeta}, j\right\rangle\right\} .
$$

Then $f_{\eta}$ clearly satisfies (i) and (ii). If $\xi<\zeta$ then, by our construction, $f_{\eta} \uparrow A_{\xi}=f_{\zeta} \uparrow A_{\xi}$, hence, as (iii) is satisfied by $f_{\zeta}$, there is $i<x$ such that $\left|f_{\eta}^{-1}\{i\} \cap A_{\xi}\right|=1$. Moreover, $f_{\eta}^{-1}\{j\} \cap A_{\zeta}=\left\{\xi_{\zeta}\right\}$, so (iii) is satisfied by $f_{\eta}$ as well.

Finally, to show that $f_{\eta}$ satisfies (iv), consider any $\gamma \geq \eta$. If we have $\left|A_{\gamma} \cap \operatorname{dom}\left(f_{\zeta} \backslash f\right)\right|<d$ then $\left|A_{\gamma} \cap \operatorname{dom}\left(f_{\eta} \backslash f\right)\right| \leq d$ holds trivially, because $\mid \operatorname{dom}\left(f_{\eta} \backslash f_{\zeta} \mid \leq 1\right.$. If, on the other hand, $\left|A_{\gamma} \cap \operatorname{dom}\left(f_{\zeta} \backslash f\right)\right|=d$ then $A_{\gamma} \in \mathcal{A}_{\zeta}$ and so $\xi_{\zeta} \notin A_{\gamma}$. Thus, in this case, $\left|A_{\gamma} \cap \operatorname{dom}\left(f_{\eta} \backslash f\right)\right|=$ $\left|A_{\gamma} \cap \operatorname{dom}\left(f_{\zeta} \backslash f\right)\right|=d$; in any case $f_{\eta}$ satisfies (iv).

Obviously, then $f_{\kappa} \in \mathcal{F}(\lambda, x)$ is a weak conflict free coloring of $\mathcal{A}$ that satisfies $f_{\kappa} \uparrow C=f$, completing the proof.

Next we prove a stepping up result for the first parameter of our new arrow relations. The proof of this will reveal why we chose to introduce this new relation.

Theorem 5.5. Let $\lambda \geq \kappa \geq \omega$ and $d, k, x \in \omega$ with $d>0$. Then

(1) $[\lambda, \kappa, d, k+d-1] \rightarrow_{w} x$ implies $\left[\lambda^{+}, \kappa, d, k\right] \rightarrow_{w} x$,

(2) $[\lambda, \kappa, d, k+d-1] \rightarrow x$ implies $\left[\lambda^{+}, \kappa, d, k\right] \rightarrow x$.

Proof of Theorem 5.5. (1). Assume that $C \subset \lambda^{+}$and the $d$-almost disjoint system $\mathcal{A} \subset\left[\lambda^{+}\right]^{\kappa}$ are such that $|A \cap C| \leq k$ for any $A \in \mathcal{A}$, moreover $f \in \mathcal{F}(C, x)$. Let $\left\langle N_{\nu}: \nu<\lambda^{+}\right\rangle$be a $\lambda^{+}$-chain of elementary submodels such that $\lambda^{+}, \mathcal{A}, C, f \in N_{1}$ and $\lambda \subset N_{1}$. 
By transfinite induction we shall define $g_{\eta} \in \mathcal{F}\left(\lambda^{+}, x\right)$ for all $\eta<\lambda^{+}$ satisfying the following inductive hypotheses.

(i) $\operatorname{dom}\left(g_{\eta}\right) \subset N_{\eta}$ and $g_{\zeta} \subset g_{\eta}$ for $\zeta<\eta$,

(ii) $g_{\eta} \uparrow C \cap N_{\eta}=f \uparrow C \cap N_{\eta}$,

(iii) $g_{\eta}$ is a weak conflict free coloring of $\mathcal{A} \cap N_{\eta}$.

Case 1. $\eta=0$.

We have to put $g_{0}=\emptyset$ because $N_{0}=\emptyset$. This works trivially for the same reason.

Case 2. $\eta$ is limit.

Then we put $g_{\eta}=\cup\left\{g_{\zeta}: \zeta<\eta\right\}$. Now, (i) and (ii) follow immediately from $N_{\eta}=\cup\left\{N_{\xi}: \xi<\eta\right\}$. To check (iii), pick $A \in \mathcal{A} \cap N_{\eta}$. There is a $\zeta<\eta$ with $A \in N_{\zeta}$ and so for every $\nu \in \eta \backslash \zeta$ there is $i_{\nu}<x$ with $\left|A \cap g_{\nu}^{-1}\left\{i_{\nu}\right\}\right|=1$. As $x$ is finite, we have an $i<x$ such that $i_{\nu}=i$ for cofinally many $\nu \in \eta$, hence $\left|A \cap g_{\eta}^{-1}\{i\}\right|=1$.

Case 3. $\eta=\zeta+1$.

Let us put $C_{\eta}=\left(C \cap N_{\eta}\right) \cup N_{\zeta}$ and $f_{\eta}=\left(f \uparrow N_{\eta}\right) \cup g_{\zeta} \in \mathcal{F}\left(C_{\eta}, x\right)$. Then for all $A \in \mathcal{A} \cap\left(N_{\eta} \backslash N_{\zeta}\right) \subset\left[\lambda^{+} \cap N_{\eta}\right]^{\kappa}$ we have

$$
\left|A \cap C_{\eta}\right| \leq|A \cap C|+\left|A \cap N_{\zeta}\right| \leq k+(d-1) .
$$

But $\left|\lambda^{+} \cap N_{\eta}\right|=\lambda$, hence we can apply $[\lambda, \kappa, d, k+d-1] \rightarrow_{w} x$ to $C_{\eta}, \mathcal{A} \cap\left(N_{\eta} \backslash N_{\zeta}\right)$, and $f_{\eta}$ to find a weak conflict free coloring $g_{\eta}$ of $\mathcal{A} \cap\left(N_{\eta} \backslash N_{\zeta}\right)$ such that $\operatorname{dom}\left(g_{\eta}\right) \subset \lambda^{+} \cap N_{\eta}$ and

$$
g_{\eta} \uparrow C_{\eta}=f_{\eta} \uparrow C_{\eta}
$$

In particular, then $g_{\zeta} \subset g_{\eta}$ and since for every $A \in \mathcal{A} \cap N_{\zeta}$ we have $A \subset N_{\zeta} \subset C_{\eta}$ we obtain that $g_{\eta}$ is a weak conflict free coloring $g_{\eta}$ of $\mathcal{A} \cap N_{\eta}$. Finally, $C \cap N_{\eta} \subset C_{\eta}$ implies $g_{\eta} \uparrow C \cap N_{\eta}=f \uparrow C \cap N_{\eta}$, which shows that $g_{\eta}$ satisfies all three inductive hypotheses and thus completes the inductive construction.

It is now obvious that the function $g=\bigcup_{\eta<\lambda^{+}} g_{\eta}$ is a weak conflict free coloring of $\mathcal{A}$ and satisfies $g\lceil C=f$, which completes the proof of (1).

(2) can be proved in a completely similar, but even simpler, manner.

Proof of Theorem 5.3. To start with, in the case $m=0$, we have to show

$$
[\kappa, \kappa, d, k] \rightarrow_{w}\left\lfloor\frac{k+d}{2}\right\rfloor+1
$$

for all natural numbers $k$ and $d$. To see this, put $x=\left\lfloor\frac{k+d}{2}\right\rfloor+1$ and note that we have $2 x \geq k+d+1$, hence $2 x-d-1 \geq k$. But then, 
applying theorem 5.4, we can conclude $[\kappa, \kappa, d, 2 x-d-1] \rightarrow_{w} x$ and hence $[\kappa, \kappa, d, k] \rightarrow_{w} x$ as well.

Now, assume that $m>0$ and theorem 5.3 has been verified for $m-1$, i.e.

$$
\left[\kappa^{+m-1}, \kappa, d, k\right] \rightarrow_{w}\left\lfloor\frac{m(d-1)+k+1}{2}\right\rfloor+1
$$

holds for all $d$ and $k$. Applying the stepping up theorem 5.5 to this formula with $k$ replaced by $k+d-1$ (and $\lambda=\kappa^{+m-1}$ ) we obtain

$$
\left[\kappa^{+m}, \kappa, d, k\right] \rightarrow_{w}\left\lfloor\frac{(m+1)(d-1)+k+1}{2}\right\rfloor+1
$$

completing the induction step from $m-1$ to $m$.

\section{A LOWER BOUND FOR $w \chi_{\mathrm{CF}}\left(\beth_{m}(\kappa), \kappa, d\right)$}

Now we know that $w \chi_{\mathrm{CF}}\left(\kappa^{+m}, \kappa, d\right)$ is finite, hence it is natural to attempt to find its exact value. The aim of this section is to execute this attempt, at least under GCH and for $d=2$ or $d$ odd. The case $m=0$ is relatively easy to deal with, using the following lemma.

Lemma 6.1. Fix a cardinal $\kappa \geq \omega$ and a natural number $t>0$. We have a procedure that assigns to any $(\kappa, \kappa, 2 t)$-system $\mathcal{F}$ another $(\kappa, \kappa, 2 t)$-system $\mathcal{F}^{*}$ in such a way that $w \chi_{\mathrm{CF}}\left(\mathcal{F}^{*}\right)>t$ holds whenever $w \chi_{\mathrm{CF}}(\mathcal{F}) \geq t$.

Proof. Given any $(\kappa, \kappa, 2 t)$-system $\mathcal{F}$, let us first choose pairwise disjoint sets $\left\{A_{n}: n<2 t\right\} \cup\left\{B_{\nu}: \nu<\kappa\right\} \subset[\kappa]^{\kappa}$. For each $n<2 t$ let $\mathcal{A}_{n} \subset\left[A_{n}\right]^{\kappa}$ be an isomorphic copy of $\mathcal{F}$. Let $\left\{X_{\nu}: \nu<\kappa\right\}$ be a one-one enumeration of the family

$$
\left\{X:|X|=2 t \text { and }\left|X \cap A_{n}\right|=1 \text { for each } n<2 t\right\}
$$

of all transversals of $\left\{A_{n}: n<2 t\right\}$. Write $C_{\nu}=B_{\nu} \cup X_{\nu}$ and let

$$
\mathcal{F}^{*}=\bigcup\left\{\mathcal{A}_{n}: n<2 t\right\} \cup\left\{C_{\nu}: \nu<\kappa\right\} .
$$

Then $\mathcal{F}^{*}$ is $2 t$-almost disjoint, because

- $\left|C_{\nu} \cap C_{\mu}\right|=\left|X_{\nu} \cap X_{\mu}\right|<2 t$ for $\nu \neq \mu$,

- $\left|C_{\nu} \cap A\right| \leq 1$ for $A \in \bigcup_{n<2 t} \mathcal{A}_{n}$.

Now, assume that $w \chi_{\mathrm{CF}}(\mathcal{F}) \geq t$ and, contrary to our claim, $h$ is a weak conflict free coloring of $\mathcal{F}^{*}$ with color set $t$. Then $w \chi_{\mathrm{CF}}\left(\mathcal{A}_{n}\right) \geq t$ implies that $h\left[A_{n}\right]=t$ for each $n<2 t$, hence for each $i<t$ there are $x_{i} \in A_{2 i}$ and $y_{i} \in A_{2 i+1}$ such that $h\left(x_{i}\right)=h\left(y_{i}\right)=i$. 
There is a $\nu<\kappa$ with $X_{\nu}=\left\{x_{i}, y_{i}: i<t\right\}$. But then $\left|h^{-1}\{i\} \cap C_{\nu}\right| \geq$ 2 for each $i<t$, and $h$ is not a weak conflict free coloring of $\mathcal{F}^{*}$, a contradiction. So, indeed, we have $w \chi_{\mathrm{CF}}\left(\mathcal{F}^{*}\right)>t$.

Theorem 6.2. For any cardinal $\kappa \geq \omega$ and integer $d \geq 2$ we have

$$
w \chi_{\mathrm{CF}}(\kappa, \kappa, d)=\left\lfloor\frac{d}{2}\right\rfloor+1 .
$$

Proof. We shall prove, by induction on $1 \leq s<\omega$, that

$$
w \chi_{\mathrm{CF}}(\kappa, \kappa, 2 s) \geq s+1 \text {. }
$$

Then, also applying theorem 5.1, we have

$$
s+1 \leq w \chi_{\mathrm{CF}}(\kappa, \kappa, 2 s) \leq w \chi_{\mathrm{CF}}(\kappa, \kappa, 2 s+1) \leq s+1,
$$

hence $\left(\circ_{s}\right)$ implies both $\left(*_{2 s}\right)$ and $\left(*_{2 s+1}\right)$.

First step: $s=1$.

Take a 2-dimensional vector space $V$ with $|V|=\kappa$ above any field of cardinality $\kappa$ and let $\mathcal{L}$ be the family of all lines (1-dimensional affine subspaces) in $V$. Then $\mathcal{L}$ is 2 -almost disjoint, hence a $(\kappa, \kappa, 2)$-system, and it trivially does not have a weak conflict free coloring with a single color. So $w \chi_{\mathrm{CF}}(\kappa, \kappa, 2) \geq 2=1+1$.

Induction step: $s \rightarrow(s+1)$.

Let $\mathcal{F}$ be a $(\kappa, \kappa, 2 s)$-system with $\mathrm{w}_{\mathrm{CF}}(\mathcal{F}) \geq s+1$. We may then apply lemma 6.1 to $\mathcal{F}$ with $t=s+1$ to conclude that the $(\kappa, \kappa, 2(s+1))$ system $\mathcal{F}^{*}$ satisfies $w \chi_{\mathrm{CF}}\left(\mathcal{F}^{*}\right) \geq t+1=(s+1)+1$.

Theorem 6.2 shows that the upper bound established in theorem 5.1 is sharp for $m=0$. We shall show next that this is also true for all $m>0$, provided that GCH holds and $d$ is odd. The following lemma plays the key role in proving this.

Lemma 6.3. For any cardinals $\lambda \geq \kappa \geq \omega$ and natural number $\ell>0$ we have a procedure assigning to any $(\lambda, \kappa, 2 \ell+1)$-system $\mathcal{F}$ $a\left(2^{\lambda}, \kappa, 2 \ell+1\right)$-system $\mathcal{F}^{*}$ so that $w \chi_{\mathrm{CF}}(\mathcal{F}) \geq \ell$ implies

$$
w \chi_{\mathrm{CF}}\left(\mathcal{F}^{*}\right) \geq w \chi_{\mathrm{CF}}(\mathcal{F})+\ell \text {. }
$$

Proof. Fix the $(\lambda, \kappa, 2 \ell+1)$-system $\mathcal{F}$ and then choose pairwise disjoint sets

$$
\left\{A_{\alpha}: \alpha<\lambda\right\} \cup\left\{C_{\delta}: \delta<2^{\lambda}\right\} \subset\left[2^{\lambda}\right]^{\lambda} .
$$

For $\alpha<\lambda$, resp. $\delta<2^{\lambda}$, let $\mathcal{A}_{\alpha} \subset\left[A_{\alpha}\right]^{\kappa}$, resp. $\mathcal{C}_{\delta} \subset\left[C_{\delta}\right]^{\kappa}$, be isomorphic copies of $\mathcal{F}$. For every $\delta<2^{\lambda}$ we also fix a one-one enumeration 
$\mathcal{C}_{\delta}=\left\{C_{\delta, i}: i<\lambda\right\}$. Let us then put

$$
\mathcal{S}=\left\{S \in\left[\bigcup_{\alpha<\lambda} A_{\alpha}\right]^{2 \ell}: \forall \alpha<\lambda\left|S \cap A_{\alpha}\right| \leq 1\right\}
$$

and $\left\{f_{\delta}: \delta<2^{\lambda}\right\}$ be an enumeration of all functions $f: \lambda \rightarrow \mathcal{S}$ that satisfy

$$
f(i) \cap f(j)=\emptyset \text { for any }\{i, j\} \in[\lambda]^{2} .
$$

Finally, let $C_{\delta, i}^{*}=C_{\delta, i} \cup f_{\delta}(i)$ and put

$$
\mathcal{F}^{*}=\bigcup_{\alpha<\lambda} \mathcal{A}_{\alpha} \cup\left\{C_{\delta, i}^{*}: \delta<2^{\lambda}, i<\lambda\right\}
$$

Claim 1. $\mathcal{F}^{*}$ is $(2 \ell+1)$-almost disjoint.

The only non-trivial case is showing $\left|C_{\delta, i}^{*} \cap C_{\delta^{\prime}, i^{\prime}}^{*}\right| \leq 2 \ell$ for $\langle\delta, i\rangle \neq$ $\left\langle\delta^{\prime}, i^{\prime}\right\rangle$. Clearly, we have

$$
C_{\delta, i}^{*} \cap C_{\delta^{\prime}, i^{\prime}}^{*} \subset\left(C_{\delta} \cap C_{\delta^{\prime}}\right) \cup\left(f_{\delta}(i) \cap f_{\delta^{\prime}}\left(i^{\prime}\right)\right) .
$$

Now, if $\delta \neq \delta^{\prime}$ then $C_{\delta} \cap C_{\delta^{\prime}}=\emptyset$ and $\left|f_{\delta}(i) \cap f_{\delta^{\prime}}\left(i^{\prime}\right)\right| \leq\left|f_{\delta}(i)\right|=2 \ell$. If, on the other hand, $\delta=\delta^{\prime}$ then $f_{\delta}(i) \cap f_{\delta}\left(i^{\prime}\right)=\emptyset$ by definition, so $\left|C_{\delta, i}^{*} \cap C_{\delta, i^{\prime}}^{*}\right|=\left|C_{\delta, i} \cap C_{\delta, i^{\prime}}\right| \leq 2 \ell$ because $\mathcal{C}_{\delta}$ is $(2 \ell+1)$-almost disjoint.

Claim 2. If $w \chi_{\mathrm{CF}}(\mathcal{F}) \geq \ell$ then $w \chi_{\mathrm{CF}}\left(\mathcal{F}^{*}\right) \geq w \chi_{\mathrm{CF}}(\mathcal{F})+\ell$.

The claim is obvious if $w \chi_{\mathrm{CF}}\left(\mathcal{F}^{*}\right) \geq \omega$, so we may assume that $w \chi_{\mathrm{CF}}\left(\mathcal{F}^{*}\right)<\omega$. Now, let $h$ be any weak conflict-free coloring of $\mathcal{F}^{*}$ with a finite color set $T$. By $w \chi_{\mathrm{CF}}\left(\mathcal{A}_{\alpha}\right) \geq \ell$, for each $\alpha<\lambda$ we have $\left|h\left[A_{\alpha}\right]\right| \geq \ell$, thus there are $I \in[\lambda]^{\lambda}$ and $M=\left\{m_{j}: j<\ell\right\} \in[T]^{\ell}$ such that $h\left[A_{\alpha}\right] \supset M$ for each $\alpha \in I$.

Let $\left\{\alpha_{\zeta, n}: \zeta<\lambda, n<2 \ell\right\}$ be distinct elements of $I$. We may then, for each $j<\ell$, pick $x_{\zeta, 2 j} \in A_{\alpha_{\zeta, 2 j}}$ and $x_{\zeta, 2 j+1} \in A_{\alpha_{\zeta, 2 j+1}}$ satisfying

$$
h\left(x_{\zeta, 2 j}\right)=h\left(x_{\zeta, 2 j+1}\right)=m_{j} .
$$

There is $\delta<2^{\lambda}$ such that $f_{\delta}(\zeta)=\left\{x_{\zeta, n}: n<2 \ell\right\}$ for all $\zeta<\lambda$, then for each $m \in M$ and $i<\lambda$ we have $\left|h^{-1}\{m\} \cap f_{\delta}(i)\right| \geq 2$. It follows that $h \uparrow\left(C_{\delta} \backslash h^{-1} M\right)$ must be a weak conflict free coloring of $\mathcal{C}_{\delta}$ with color set $T \backslash M$, showing that $|T \backslash M| \geq w \chi_{\mathrm{CF}}(\mathcal{F})$, hence $|T| \geq w \chi_{\mathrm{CF}}(\mathcal{F})+\ell$, completing the proof.

Theorem 6.4. For any $\kappa \geq \omega$ and $m, \ell \in \omega$ with $\ell>0$ we have

$$
w \chi_{\mathrm{CF}}\left(\beth_{m}(\kappa), \kappa, 2 \ell+1\right) \geq(m+1) \cdot \ell+1 .
$$

Proof. By Theorem 6.2, we have $w \chi_{\mathrm{CF}}(\kappa, \kappa, 2 \ell+1)=\ell+1$. So we may simply apply theorem $6.4 \mathrm{~m}$ times to obtain the result. 
As an immediate consequence of theorems 4.16.4 we obtain the following result.

Theorem 6.5. For every infinite cardinal $\kappa$ and natural number $d>1$ we have

$$
\chi_{\mathrm{CF}}\left(\beth_{\omega}(\kappa), \kappa, d\right)=\omega .
$$

¿From theorems 5.1 and 6.4 we may immediately deduce the promised exact value of $w \chi_{\mathrm{CF}}\left(\kappa^{+m}, \kappa, 2 \ell+1\right)$ under $\mathrm{GCH}$.

Corollary 6.6. If $G C H$ holds then for any cardinal $\kappa \geq \omega$ and integers $m \geq 0, \ell>0$ we have

$$
w \chi_{\mathrm{CF}}\left(\kappa^{+m}, \kappa, 2 \ell+1\right)=(m+1) \cdot \ell+1 .
$$

We do not know, in general, if an exact formula like this can be obtained for $w \chi_{\mathrm{CF}}\left(\kappa^{+m}, \kappa, 2 \ell\right)$, but we do know this in the simplest case $\ell=1$. The key to this is again a "lift up" lemma in the spirit of lemmas 6.1 and 6.3.

Lemma 6.7. For any $\lambda \geq \kappa \geq \omega$, we can assign to every $(\lambda, \kappa, 2)$ system $\mathcal{F}$ a $\left(2^{2^{\lambda}}, \kappa, 2\right)$-system $\mathcal{F}^{*}$ so that if $w \chi_{\mathrm{CF}}(\mathcal{F})$ is finite then

$$
w \chi_{\mathrm{CF}}\left(\mathcal{F}^{*}\right)>w \chi_{\mathrm{CF}}(\mathcal{F}) \text {. }
$$

Proof. Let $\mathcal{F}$ be any $(\lambda, \kappa, 2)$-system and, to start with, fix pairwise disjoint sets

$$
\begin{aligned}
& \left\{A_{\delta}: \delta<2^{\lambda}\right\} \cup\left\{B_{\eta, \alpha}: \eta<2^{2^{\lambda}}, \alpha<\lambda\right\} \cup \\
& \quad\left\{C_{\eta, \delta}: \eta<2^{2^{\lambda}}, \delta<2^{\lambda}\right\} \subset\left[2^{2^{\lambda}}\right]^{\lambda} .
\end{aligned}
$$

For every $\delta<2^{\lambda}$ let $\mathcal{A}_{\delta} \subset\left[A_{\zeta}\right]^{\kappa}$ be an isomorphic copy of $\mathcal{F}$ and define similarly $\mathcal{B}_{\eta, \alpha} \subset\left[B_{\eta, \alpha}\right]^{\kappa}$ and $\mathcal{C}_{\eta, \delta} \subset\left[C_{\eta, \delta}\right]^{\kappa}$. We also enumerate, without repetitions, each $\mathcal{C}_{\eta, \delta}$ as $\left\{C_{\eta, \delta, i}: i<\lambda\right\}$.

Let us put $A=\cup\left\{A_{\delta}: \delta<2^{\lambda}\right\}$ and $B_{\eta}=\cup\left\{B_{\eta, \alpha}: \alpha<\lambda\right\}$ for each $\eta<2^{2^{\lambda}}$. Then enumerate the injective functions $f: 2^{\lambda} \times \lambda \rightarrow A$ as $\left\{f_{\eta}: \eta<2^{2^{\lambda}}\right\}$ and, for any $\eta<2^{2^{\lambda}}$, enumerate the injective functions $g: \lambda \rightarrow B_{\eta}$ as $\left\{g_{\eta, \delta}: \delta<2^{\lambda}\right\}$. Finally, let

$$
C_{\eta, \delta, i}^{*}=C_{\eta, \delta, i} \cup\left\{f_{\eta}(\delta, i), g_{\eta, \delta}(i)\right\}
$$

and put

$$
\begin{aligned}
& \mathcal{F}^{*}=\bigcup\left\{\mathcal{A}_{\delta}: \delta<2^{\lambda}\right\} \cup \bigcup\left\{\mathcal{B}_{\eta, \alpha}:\langle\eta, \alpha\rangle\right.\left.\in 2^{2^{\lambda}} \times \lambda\right\} \cup \\
& \cup\left\{C_{\eta, \delta, i}^{*}:\langle\eta, \delta, i\rangle \in 2^{2^{\lambda}} \times 2^{\lambda} \times \lambda\right\} .
\end{aligned}
$$

Claim 1. $\mathcal{F}^{*}$ is 2-almost disjoint. 
The only non-trivial task is to show that $\left|C_{\eta, \delta, i}^{*} \cap C_{\eta^{\prime}, \delta^{\prime}, i^{\prime}}^{*}\right| \leq 1$ for $\langle\eta, \delta, i\rangle \neq\left\langle\eta^{\prime}, \delta^{\prime}, i^{\prime}\right\rangle$. Clearly, we have

$$
\begin{aligned}
C_{\eta, \delta, i}^{*} \cap C_{\eta^{\prime}, \delta^{\prime}, i^{\prime}}^{*} & \subset\left(C_{\eta, \delta, i} \cap C_{\eta^{\prime}, \delta^{\prime}, i^{\prime}}\right) \cup \\
\cup\left(\left\{f_{\eta}(\delta, i)\right\}\right. & \left.\left.\cap\left\{f_{\eta^{\prime}}\left(\delta^{\prime}, i^{\prime}\right)\right\}\right) \cup\left(\left\{g_{\eta, \delta}(i)\right\} \cap\left\{g_{\eta^{\prime}, \delta^{\prime}}\left(i^{\prime}\right)\right\}\right)\right) .
\end{aligned}
$$

If $\eta \neq \eta^{\prime}$ then $C_{\eta, \delta} \cap C_{\eta^{\prime}, \delta^{\prime}}=\emptyset$, and $\left\{g_{\eta, \delta}(i)\right\} \cap\left\{g_{\eta^{\prime}, \delta^{\prime}}\left(i^{\prime}\right)\right\} \subset B_{\eta} \cap B_{\eta^{\prime}}=\emptyset$, hence

$$
C_{\eta, \delta, i}^{*} \cap C_{\eta^{\prime}, \delta^{\prime}, i^{\prime}}^{*} \subset\left\{f_{\eta}(\delta, i)\right\} \cap\left\{f_{\eta^{\prime}}\left(\delta^{\prime}, i^{\prime}\right)\right\} .
$$

If $\eta=\eta^{\prime}$ and $\delta \neq \delta^{\prime}$ then $C_{\eta, \delta} \cap C_{\eta, \delta^{\prime}}=\emptyset$, and $f_{\eta}(\delta, i) \neq f_{\eta}\left(\delta^{\prime}, i^{\prime}\right)$ because $f_{\eta}$ is injective, hence

$$
C_{\eta, \delta, i}^{*} \cap C_{\eta^{\prime}, \delta^{\prime}, i^{\prime}}^{*} \subset\left\{g_{\eta, \delta}(i)\right\} \cap\left\{g_{\eta^{\prime}, \delta^{\prime}}\left(i^{\prime}\right)\right\} .
$$

Finally, if $\eta=\eta^{\prime}, \delta=\delta^{\prime}$, and $i \neq i^{\prime}$ then $f_{\eta}(\delta, i) \neq f_{\eta}\left(\delta, i^{\prime}\right)$ and $g_{\eta, \delta}(i) \neq g_{\eta, \delta}\left(i^{\prime}\right)$ because $g_{\eta, \delta}$ is also injective, and so

$$
\left|C_{\eta, \delta, i}^{*} \cap C_{\eta^{\prime}, \delta^{\prime}, i^{\prime}}^{*}\right|=\left|C_{\eta, \delta, i} \cap C_{\eta^{\prime}, \delta^{\prime}, i^{\prime}}\right| \leq 1 \text {. }
$$

Claim 2. $w \chi_{\mathrm{CF}}\left(\mathcal{F}^{*}\right)>w \chi_{\mathrm{CF}}(\mathcal{F})$ if the latter is finite.

Assume that $w \chi_{\mathrm{CF}}(\mathcal{F})=k<\omega$ and, contrary to our claim, $h$ is a weak conflict-free coloring of $\mathcal{F}^{*}$ with $\operatorname{ran}(h)=k$. Then, for each $\delta<2^{\lambda}$, the equality $w \chi_{\mathrm{CF}}\left(\mathcal{A}_{\delta}\right)=k$ implies that there is $a_{\delta} \in A_{\delta}$ with $h\left(a_{\delta}\right)=0$. Since $\left|\left\{a_{\delta}: \delta<2^{\lambda}\right\}\right|=2^{\lambda}$, there is an $\eta<2^{2^{\lambda}}$ with $\operatorname{ran}\left(f_{\eta}\right)=\left\{a_{\delta}: \delta<2^{\lambda}\right\} \subset h^{-1}\{0\}$.

Fix this $\eta$ and then apply $w \chi_{\mathrm{CF}}\left(\mathcal{B}_{\eta, \alpha}\right)=k$ to find, for each $\alpha<\lambda$, some $b_{\alpha} \in B_{\eta, \alpha}$ with $h\left(b_{\alpha}\right)=0$. Again, we have $\left|\left\{b_{\alpha}: \alpha<\lambda\right\}\right|=\lambda$, hence there is a $\delta<2^{\lambda}$ with $\operatorname{ran}\left(g_{\eta, \delta}\right)=\left\{b_{\alpha}: \alpha<\lambda\right\} \subset h^{-1}\{0\}$.

But then for each $i<\lambda$ we have $\left\{f_{\eta}(\delta, i), g_{\eta, \delta}(i)\right\} \subset h^{-1}\{0\}$, consequently $h \uparrow\left(C_{\eta, \delta} \backslash h^{-1}\{0\}\right)$ must be a weak conflict free coloring of $\mathcal{C}_{\eta, \delta}$ with $k-1$ colors, a contradiction. This contradiction proves Claim 2 and completes the proof of the lemma.

Theorem 6.8. For any $\kappa \geq \omega$ and $m \in \omega$ we have

$$
w \chi_{\mathrm{CF}}\left(\beth_{m}(\kappa), \kappa, 2\right) \geq\left\lfloor\frac{m}{2}\right\rfloor+2 .
$$

Proof. By theorem 6.2 this is true for $m=0$ and $m=1$. Moreover, if we assume $w \chi_{\mathrm{CF}}\left(\beth_{m}(\kappa), \kappa, 2\right) \geq\left\lfloor\frac{m}{2}\right\rfloor+2$ then, applying lemma 6.7 with $\lambda=\beth_{m}(\kappa)$, we obtain

$$
w \chi_{\mathrm{CF}}\left(\beth_{m+2}(\kappa), \kappa, 2\right) \geq\left\lfloor\frac{m}{2}\right\rfloor+2+1=\left\lfloor\frac{m+2}{2}\right\rfloor+2 .
$$

Thus the theorem follows by a straight-forward induction.

Comparing this with theorem 5.1 we get the following result. 
Corollary 6.9. For $\kappa \geq \omega$ and $m \in \omega$, the equality $\beth_{m}(\kappa)=\kappa^{+m}$ implies

$$
w \chi_{\mathrm{CF}}\left(\kappa^{+m}, \kappa, 2\right)=\left\lfloor\frac{m}{2}\right\rfloor+2 .
$$

\section{Attempts to Compute $\chi_{\mathrm{CF}}\left(\omega_{k}, \omega, 2\right)$}

In the previous section we succeeded in computing the exact value of $w \chi_{\mathrm{CF}}\left(\kappa^{+m}, \kappa, d\right)$ in a lot of cases, at least under GCH. As we have

$$
w \chi_{\mathrm{CF}}(\lambda, \kappa, d) \leq \chi_{\mathrm{CF}}(\lambda, \kappa, d) \leq w \chi_{\mathrm{CF}}(\lambda, \kappa, d)+1,
$$

this gives us a lot of information about $\chi_{\mathrm{CF}}\left(\kappa^{+m}, \kappa, d\right)$ as well. But can we find the exact value of $\chi_{\mathrm{CF}}\left(\kappa^{+m}, \kappa, d\right)$, or even just of $\chi_{\mathrm{CF}}\left(\omega_{m}, \omega, d\right)$, say under GCH and for many values of $m$ and $d$ ? This turned out to be a very hard problem that we address in the present section, admittedly with only rather meager results. There is no problem in the simplest possible case: $m \leq 1$ and $d=2$.

Proposition 7.1. $\chi_{\mathrm{CF}}(\kappa, \kappa, 2)=\chi_{\mathrm{CF}}\left(\kappa^{+}, \kappa, 2\right)=3$ for all $\kappa \geq \omega$.

Proof. First, by theorem 5.1, we have

$$
\chi_{\mathrm{CF}}(\kappa, \kappa, 2) \leq \chi_{\mathrm{CF}}\left(\kappa^{+}, \kappa, 2\right) \leq 3 .
$$

We have seen in the proof of theorem 6.2 that if $V$ is any 2-dimensional vector space with $|V|=\kappa$ above any field of cardinality $\kappa$, then the $(\kappa, \kappa, 2)$-system $\mathcal{L}$ of all lines in $V$ satisfies

$$
w \chi_{\mathrm{CF}}(\mathcal{L})=w \chi_{\mathrm{CF}}(\kappa, \kappa, 2)=2 .
$$

Consequently, we shall be done if we can show that $\mathcal{L}$ does not have a conflict free coloring with 2 colors.

Assume, on the contrary, that $f: V \rightarrow 2$ is a CF-coloring of $\mathcal{L}$ and write $C_{i}=f^{-1}\{i\}$ for $i \in 2$. Since $\left|C_{i} \cap L\right| \geq 1$ for each line $L$ and color $i<2$, neither $C_{i}$ is collinear, i.e. $C_{i} \not \subset L$ for any $i<2$ and for any line $L$. Thus there are four lines $\left\{K_{i}^{j}: i, j<2\right\} \subset \mathcal{L}$ such that $\left|C_{i} \cap K_{i}^{j}\right| \geq 2$ for all $i, j<2$. Since $f$ is a CF-coloring, for any $i, j<2$ we have a point $P_{i}^{j}$ with $K_{i}^{j} \cap C_{1-i}=\left\{P_{i}^{j}\right\}$.

There is a line $L$ that intersects each $K_{i}^{j}$ in distinct points which are all different from the points $P_{i}^{j}$. Then $\left|L \cap C_{i}\right| \geq 2$ for $i<2$, hence $f$ is not a $\mathrm{CF}$-coloring of $\mathcal{L}$, a contradiction.

What can we say about $\chi_{\mathrm{CF}}\left(\omega_{m}, \omega, 2\right)$ for $m>1$ ? If $\beth_{m}=\omega_{m}$, in particular under GCH, from corollary 6.9, we have, for any $m<\omega$,

$$
\left\lfloor\frac{m}{2}\right\rfloor+2 \leq \chi_{\mathrm{CF}}\left(\omega_{m}, \omega, 2\right) \leq\left\lfloor\frac{m}{2}\right\rfloor+3,
$$


hence, in particular,

$$
3 \leq \chi_{\mathrm{CF}}\left(\omega_{2}, \omega, 2\right) \leq \chi_{\mathrm{CF}}\left(\omega_{3}, \omega, 2\right) \leq 4
$$

We actually do not know the exact value of $\chi_{\mathrm{CF}}\left(\omega_{2}, \omega, 2\right)$ even under $\mathrm{GCH}$, but we can reformulate the problem in terms of the strict fiveparameter arrow relation that was introduced in definition 5.2. One direction of this works in ZFC.

Theorem 7.2. If $[\kappa, \kappa, 2,2] \rightarrow 3$ then $\chi_{\mathrm{CF}}\left(\kappa^{++}, \kappa, 2\right)=3$.

Proof. Starting with the relation $[\kappa, \kappa, 2,2] \rightarrow 3$ and applying theorem 5.5 (2) twice we obtain $\left[\kappa^{++}, \kappa, 2,0\right] \rightarrow 3$ which, of course, is just $\left[\kappa^{++}, \kappa, 2\right] \rightarrow 3$, and hence, together with $\chi_{\mathrm{CF}}(\kappa, \kappa, 2)=3$, implies $\chi_{\mathrm{CF}}\left(\kappa^{++}, \kappa, 2\right)=3$.

To go in the opposite direction, we first need the following result concerning the relation $[\lambda, \kappa, 2, k] \rightarrow x$.

Lemma 7.3. If $[\lambda, \kappa, 2, k] \not \rightarrow x$ then this can be witnessed by a $(\lambda, \kappa, 2)$ system $\mathcal{X}=\left\{X_{i}: i<\lambda\right\} \subset[\lambda]^{\kappa}$ and a map $c \in \mathcal{F}(\lambda, x)$ such that

$$
Y=\operatorname{dom}(c)=\cup\left\{Y_{i}: i<\lambda\right\},
$$

where $X_{i} \cap Y \subset Y_{i} \in[Y]^{k}$ for each $i<\lambda$ and the $k$-element sets $Y_{i}$ are pairwise disjoint.

Proof. Fix an arbitrary $(\lambda, \kappa, 2)$-system $\mathcal{X}=\left\{X_{i}: i<\lambda\right\} \subset[\lambda]^{\kappa}$ and a map $c \in \mathcal{F}(\lambda, x)$ that witnesses $[\lambda, \kappa, 2, k] \not \rightarrow x$. For each $y \in Y$ consider the set $I_{y}=\left\{i \in \lambda: X_{i} \cap Y \neq \emptyset\right\}$ and if $\left|I_{y}\right|>1$ then, for each $i \in I_{y}$ replace $y$ in $X_{i}$ by the pair $\langle y, i\rangle$ and "blow up" $y$ in $Y$ to $I_{y} \times\{y\}$. Having done this for all $y \in Y$ let us denote the "new" $X_{i}$ by $X_{i}^{\prime}$ and the "new" $Y$ by $Y^{\prime}$. Also define the "new" function $c^{\prime}$ on $Y^{\prime}$ by the rule $c^{\prime}(\langle y, i\rangle)=c(y)$. We may then add, if necessary, completely new elements to $Y^{\prime}$ (and extend $c^{\prime}$ to them arbitrarily) to obtain the pairwise disjoint $k$-element sets $Y_{i} \supset X_{i}^{\prime} \cap Y^{\prime}$ forming a partition of $Y^{\prime}$.

It is easy to check that the $(\lambda, \kappa, 2)$-system $\mathcal{X}^{\prime}=\left\{X_{i}^{\prime}: i<\lambda\right\}$ and the map $c^{\prime}$, that now are of the desired form, also witness $[\lambda, \kappa, 2, k] \nrightarrow \rightarrow x$.

Theorem 7.4. For any $\lambda \geq \kappa \geq \omega>k$,

$$
\chi_{\mathrm{CF}}(\lambda, \kappa, 2)=\chi_{\mathrm{CF}}\left(\beth_{k}(\lambda), \kappa, 2\right)=x<\omega
$$

implies $[\lambda, \kappa, 2, k] \rightarrow x$.

Proof. By the previous result, to conclude $[\lambda, \kappa, 2, k] \rightarrow x$, it suffices to show the existence of a conflict free coloring of $\mathcal{X}$ that extends $c$ for any $(\lambda, \kappa, 2)$-system $\mathcal{X}=\left\{X_{i}: i<\lambda\right\} \subset[\lambda]^{\kappa}$ and partial map 
$c \in \mathcal{F}(\lambda, x)$ satisfying the conditions of lemma 7.3. That is, we may assume having a partition $\left\{Y_{i}: i<\lambda\right\}$ of $\operatorname{dom}(c)=Y$ into disjoint $k$-element sets such that $X_{i} \cap Y \subset Y_{i}$ for all $i<\lambda$. For each $i<\lambda$ we write $Y_{i}=\left\{y_{i}^{j}: 1 \leq j \leq k\right\}$. By $\chi_{\mathrm{CF}}(\lambda, \kappa, 2)=x$, we can fix a $(\lambda, \kappa, 2)$-system $\mathcal{F}$ with $\chi_{\mathrm{CF}}(\mathcal{F})=x$.

We now introduce some notation. For any $j$ we write $\beth_{j}(\lambda)=\lambda_{j}$ (so, in particular, $\lambda_{0}=\lambda$ ) and put $\Pi=\lambda_{k} \times \lambda_{k-1} \times \ldots \times \lambda_{0}$. For each $j \leq k$ we shall also write $\Pi^{j}=\lambda_{k} \times \ldots \times \lambda_{j+1} \times \lambda_{j-1} \times \ldots \times \lambda_{0}$, that is the members of $\Pi^{j}$ are obtained from the members of $\Pi$ by deleting their $j$-coordinate.

Next we choose pairwise disjoint sets $\left\{A_{\sigma}^{j}: j \leq k, \sigma \in \Pi^{j}\right\}$ of size $\lambda$, and for every $j$ with $1 \leq j \leq k$ and $\sigma \in \Pi^{j}$ we let $\mathcal{A}_{\sigma}^{j}$ be a copy of $\mathcal{F}$ on $A_{\sigma}^{j}$.

For fixed $j$ with $1 \leq j \leq k$ and $\varrho \in \lambda_{k} \times \ldots \times \lambda_{j+1}$, consider the family $\mathbb{F}_{\varrho}^{j}$ of all functions $f$ such that $\operatorname{dom}(f)=\lambda_{j-1} \times \ldots \times \lambda_{0}$ and $f(\eta) \in A_{\varrho \curvearrowright \eta}^{j}$ for all $\eta \in \lambda_{j-1} \times \ldots \times \lambda_{0}$. Then $\left|\mathbb{F}_{\varrho}^{j}\right|=\lambda_{j}$, hence for every $j$ with $1 \leq j \leq k$ there is a function $f^{j}$ with $\operatorname{dom}\left(f^{j}\right)=\Pi$ and having the property that, if we fix $\varrho \in \lambda_{k} \times \ldots \times \lambda_{j+1}$, then the functions $\eta \mapsto f^{j}(\varrho \frown\langle\xi\rangle \frown \eta)$ enumerate $\mathbb{F}_{\varrho}^{j}$ in a one-one manner, as $\xi$ ranges over $\lambda_{j}$.

For any $\sigma \in \Pi^{0}$ we put

$$
B_{\sigma}^{0}=A_{\sigma}^{0} \cup\left\{f^{j}(\sigma \frown\langle i\rangle): 1 \leq j \leq k \text { and } i<\lambda\right\} .
$$

Then, as $|\lambda \backslash Y|=\lambda$, we may fix a bijection $h_{\sigma}: \lambda \rightarrow B_{\sigma}^{0}$ such that

$$
h_{\sigma}[\lambda \backslash Y]=A_{\sigma}^{0} \text { and } h_{\sigma}\left(y_{i}^{j}\right)=f^{j}(\sigma \frown\langle i\rangle)
$$

for any $1 \leq j \leq k$ and $i<\lambda$. Now, if $\tau \in \Pi$ with $\tau=\sigma \frown<i>$ then we set $B_{\tau}=h_{\sigma}\left[X_{i}\right]$.

We claim that the family

$$
\mathcal{A}=\bigcup\left\{\mathcal{A}_{\sigma}^{j}: 1 \leq j \leq k \text { and } \sigma \in \Pi^{j}\right\} \cup\left\{B_{\tau}: \tau \in \Pi\right\}
$$

is 2-almost disjoint. Here the only problematic task is to show that $\left|B_{\tau} \cap B_{\tau^{\prime}}\right| \leq 1$ for two distinct members, $\tau=\left\langle\xi_{k}, \ldots, \xi_{1}, i\right\rangle$ and $\tau^{\prime}=$ $\left\langle\xi_{k}^{\prime}, \ldots, \xi_{1}^{\prime}, i^{\prime}\right\rangle$, of $\Pi$. Let $\sigma=\left\langle\xi_{k}, \ldots, \xi_{1}\right\rangle$ and $\sigma^{\prime}=\left\langle\xi_{k}^{\prime}, \ldots, \xi_{1}^{\prime}\right\rangle$. If $\sigma \neq \sigma^{\prime}$ and $j \geq 1$ is maximal such that $\xi_{j} \neq \xi_{j}^{\prime}$, then we have $B_{\tau} \cap B_{\tau^{\prime}} \subset$ $\left\{f^{j}(\tau)\right\} \cap\left\{f^{j}\left(\tau^{\prime}\right)\right\}$. If, however, $\sigma=\sigma^{\prime}$ then $i \neq i^{\prime}$ and

$$
B_{\tau} \cap B_{\tau^{\prime}}=h_{\sigma}\left[X_{i}\right] \cap h_{\sigma}\left[X_{i^{\prime}}\right]=h_{\sigma}\left[X_{i} \cap X_{i^{\prime}}\right],
$$

hence we are done because $\mathcal{X}$ is 2-almost disjoint.

Thus $\mathcal{A}$ is a $\left(\lambda_{k}, \kappa, 2\right)$-system and so, by our assumption, it has a conflict free coloring $d: \cup \mathcal{A} \rightarrow x$. Our choice of $\mathcal{A}_{\varrho}^{j}$ implies that, for every $j$ with $1 \leq j \leq k$ and $\varrho \in \Pi^{j}$, we have $d\left[A_{\varrho}^{j}\right]=x$. It follows 
that there is a function $f \in \mathbb{F}_{\emptyset}^{k}$ which satisfies $d(f(\varrho))=c\left(y_{i}^{k}\right)$ for all $\varrho \in \Pi^{k}$, where $i$ is the last (0) coordinate of $\varrho$, and there is an ordinal $\xi_{k}<\lambda_{k}$ for which we have $f(\varrho)=f^{k}\left(\left\langle\xi_{k}\right\rangle \frown \varrho\right)$ for all $\varrho \in \Pi^{k}$.

Repeating this procedure "downward", step by step, we arrive at a sequence $\sigma=\left\langle\xi_{k}, \ldots, \xi_{1}\right\rangle \in \Pi^{0}$ which, for any $j$ with $1 \leq j \leq k$ and $i<\lambda$, satisfies the equality

$$
d\left(f^{j}(\sigma \frown\langle i\rangle)\right)=c\left(y_{i}^{j}\right) .
$$

But recall that we have $h_{\sigma}\left(y_{i}^{j}\right)=f^{j}(\sigma \frown\langle i\rangle)$ by definition, hence the composition $d \circ h_{\sigma}$ is a conflict free coloring of $\mathcal{X}$ which extends $c$, completing our proof of $[\lambda, \kappa, 2, k] \rightarrow x$.

Corollary 7.5. For every infinite cardinal $\kappa, \chi_{\mathrm{CF}}\left(\beth_{2}(\kappa), \kappa, 2\right)=3$ implies $[\kappa, \kappa, 2,2] \rightarrow 3$. Consequently, if $\beth_{2}(\kappa)=\kappa^{++}$, in particular under $G C H,[\kappa, \kappa, 2,2] \rightarrow 3$ is equivalent to $\chi_{\mathrm{CF}}\left(\kappa^{++}, \kappa, 2\right)=3$.

Our next aim is to show that $\chi_{\mathrm{CF}}\left(\omega_{3}, \omega, 2\right)=4$ under GCH. This will follow from the ZFC result $\chi_{\mathrm{CF}}\left(\beth_{3}, \omega, 2\right) \geq 4$ that, in turn, follows from the negative relation $[\omega, \omega, 2,3] \not \rightarrow 3$. To prove the latter, we need the following technical lemma.

Lemma 7.6. There are a finite 2-almost disjoint family $\mathcal{A}$ of countably infinite sets, a finite set $C$, and a function $c: C \rightarrow 3$ such that

(1) $|A \cap C|=4$ for each $A \in \mathcal{A}$,

(2) the sets $\{A \cap C: A \in \mathcal{A}\}$ are pairwise disjoint,

(3) c can not be extended to a conflict free coloring of $\mathcal{A}$ with 3 colors.

Proof. For $\{a, b\} \in[\mathbb{R}]^{2}$ let $L_{a, b}$ be the line in $\mathbb{R}^{2}$ which contains $a$ and $b$ and put $E_{a, b}=L_{a, b} \cap \mathbb{Z}^{2}$. We then put

$$
\mathcal{A}=\left\{E_{a, b}:\{a, b\} \in[4 \times 6]^{2}\right\} .
$$

Let $C \subset \cup \mathcal{A} \backslash(4 \times 6)$ be any finite set that satisfies (1) and (2).

Write $V_{i}=E_{\langle i, 0\rangle,\langle i, 1\rangle}$ for $i<4$ and $H_{j}=E_{\langle 0, j\rangle,\langle 1, j\rangle}$ for $j<6$. Define $c: C \rightarrow 3$ in such a way that if $C_{i}=c^{-1}\{i\}$ for $i<3$, then we have

(a) for each $i<4$

$$
\left|C_{0} \cap V_{i}\right|=\left|C_{1} \cap V_{i}\right|=2
$$

(b) for each $j<6$

$$
\left|C_{1} \cap H_{j}\right|=\left|C_{2} \cap H_{j}\right|=2
$$

(c) for each $i \neq i^{\prime}<4$ and $j \neq j^{\prime}<6$

$$
\left|C_{0} \cap E_{\langle i, j\rangle,\left\langle i^{\prime}, j^{\prime}\right\rangle}\right|=\left|C_{2} \cap E_{\langle i, j\rangle,\left\langle i^{\prime}, j^{\prime}\right\rangle}\right|=2
$$


Assume that $f: \cup \mathcal{A} \rightarrow 3$ is a conflict free coloring of $\mathcal{A}$ with $c \subset f$. Then, by (a), for each $i<4$ there is exactly one $x_{i} \in V_{i}$ such that $f\left(x_{i}\right)=2$. Since $6-4=2$ there are $j \neq j^{\prime}<6$ such that

$$
\left\{x_{i}: i<4\right\} \cap\left(H_{j} \cup H_{j^{\prime}}\right)=\emptyset \text {. }
$$

By (b), there are unique $y_{j} \in H_{j}$ and $y_{j^{\prime}} \in H_{j^{\prime}}$, respectively, such that $f\left(y_{j}\right)=f\left(y_{j^{\prime}}\right)=0$. Since $4-2=2$ there are $i \neq i^{\prime}<4$ such that

$$
\left\{y_{j}, y_{j^{\prime}}\right\} \cap\left(V_{i} \cup V_{i^{\prime}}\right)=\emptyset .
$$

Let $a=\langle i, j\rangle$ and $b=\left\langle i^{\prime} j^{\prime}\right\rangle$. Then $a \neq x_{i}$ implies $f(a) \neq 2$ and similarly, $a \neq y_{j}$ implies $f(a) \neq 0$, hence $f(a)=1$. Similarly, we have $f(b)=1$. But, as $a, b \in E_{a, b}$ and (c) holds, we have $\left|E_{a, b} \cap f^{-1}\{i\}\right|>1$ for each $i<3$, which is a contradiction.

Theorem 7.7. $[\omega, \omega, 2,3] \not \rightarrow 3$.

Proof. We shall construct a 2-almost disjoint family $\mathcal{H} \subset[H]^{\omega}$ for a countable set $H$, a subset $K \subset H$, and a function $d: K \rightarrow 3$ such that

(1) $|H \cap K| \leq 3$ for each $H \in \mathcal{H}$,

(2) $d$ can not be extended to a conflict free coloring of $\mathcal{H}$ with 3 colors.

We first choose, using $\chi_{\mathrm{CF}}(\omega, \omega, 2)=3$, a 2-almost disjoint family $\mathcal{B} \subset[\omega]^{\omega}$ such that

(10) if $f: \omega \rightarrow 3$ is any conflict-free coloring of $\mathcal{B}$

$$
\text { then } f^{-1}\{i\} \text { is infinite for each } i<3 \text {. }
$$

(Let $\left\{A_{n}: n<\omega\right\}$ be a partition of $\omega$ into infinite sets and $\mathcal{B}_{n} \subset\left[A_{n}\right]^{\omega}$ be a copy of a family witnessing $\chi_{\mathrm{CF}}(\omega, \omega, 2)=3$. Then $\mathcal{B}=\cup_{n<\omega} \mathcal{B}_{n}$ clearly satisfies (10).)

Fix a countable set $X$, a finite family $\mathcal{A} \subset[X]^{\omega}$, a finite set $C \subset X$, and a function $c: C \rightarrow 3$ as in Lemma 7.6. $D \subset C$ be such that $|A \cap D|=1$ for each $A \in \mathcal{A}$.

Let $\mathcal{G}$ denote the collection of all injective functions $g: D \stackrel{1-1}{\longrightarrow} \omega$ and $\left\{H_{g}: g \in \mathcal{G}\right\}$ be disjoint countably infinite sets with $H_{g} \cap \omega=\emptyset$. For each $g \in \mathcal{G}$ fix a bijection $h_{g}^{\prime}:(X \backslash D) \rightarrow H_{g}$ and put $h_{g}=g \cup h_{g}^{\prime}$.

Let us then define

$$
\begin{gathered}
H=\omega \cup \bigcup\left\{H_{g}: g \in \mathcal{G}\right\}, \\
\mathcal{H}=\mathcal{B} \cup\left\{h_{g}[A]: A \in \mathcal{A}, g \in \mathcal{G}\right\}, \\
K=\cup\left\{h_{g}[C \backslash D]: g \in \mathcal{G}\right\},
\end{gathered}
$$

and, finally, define $d: K \rightarrow 3$ as follows:

(14) if $k=h_{g}(x)$ for some $x \in C \backslash D$ and $g \in \mathcal{G}$, then $d(k)=c(x)$. 
We claim that $H, \mathcal{H}, K$, and $d$ are as required, that is satisfy (1) and (2). Of course, only (2) needs to be checked.

Assume, on the contrary, that $f: H \rightarrow 3$ is a conflict-free coloring for $\mathcal{H}$ with $d \subset f$. Using (10) we may find an injective function $g: D \rightarrow \omega$ such that for each $x \in D$ we have

$$
f(g(x))=c(x) .
$$

Let us now define $F: \omega \rightarrow 3$ by $F(x)=f\left(h_{g}(x)\right)$. Since $f$ is a conflict free coloring of $\left\{h_{g}[A]: A \in \mathcal{A}\right\} \subset \mathcal{H}$ and $h_{g}$ is a bijection, $F$ is a conflict free coloring of $\mathcal{A}$.

If $x \in D$ then $F(x)=f\left(h_{g}(x)\right)=f(g(x))=c(x)$ by (15) and if $x \in C \backslash D$ then $F(x)=f\left(h_{g}(x)\right)=d\left(h_{g}(x)\right)=c(x)$ by (14), hence $c \subset F$. But this contradicts the choice of $\mathcal{A}$, which proves that $H, K$, $\mathcal{H}$, and $d$ really satisfy conditions (1) and (2).

Corollary 7.8. $\chi_{\mathrm{CF}}\left(\beth_{3}, \omega, 2\right) \geq 4$. Consequently, if $\beth_{3}=\omega_{3}$ then $\chi_{\mathrm{CF}}\left(\omega_{3}, \omega, 2\right)=4$.

Problem 7.1. Is $\chi_{\mathrm{CF}}\left(\omega_{2}, \omega, 2\right)=4$ provable under $G C H$ ?

$$
\text { PART III. ThE CASE } \lambda \geq \kappa \geq \omega=\mu
$$

\section{Consistent upper Bounds FOR $\chi_{\mathrm{CF}}(\lambda, \kappa, \omega)$}

We start by pointing out that $\chi_{\mathrm{CF}}(\lambda, \kappa, \omega)$ is always infinite. This follows immediately from the next proposition because $\chi_{\mathrm{CF}}(\lambda, \kappa, \omega)$ is increasing in its first parameter.

Proposition 8.1. For every infinite cardinal $\kappa$ we have

$$
\chi_{\mathrm{CF}}(\kappa, \kappa, \omega) \geq \omega \text {. }
$$

Proof. By theorem 6.2, for every $d \in \omega \backslash 2$ there is a $(\kappa, \kappa, d)$-system $\mathcal{A}_{d}$ such that

$$
\chi_{\mathrm{CF}}\left(\mathcal{A}_{d}\right) \geq w \chi_{\mathrm{CF}}\left(\mathcal{A}_{d}\right)=\left\lfloor\frac{d}{2}\right\rfloor+1 .
$$

But clearly if $\mathcal{A}$ is the union of $\left\{\mathcal{A}_{d}: d \in \omega \backslash 2\right\}$ (taken on disjoint underlying sets) then $\mathcal{A}$ is a $(\kappa, \kappa, \omega)$-system with $\chi_{\mathrm{CF}}(\mathcal{A}) \geq \omega$.

The main aim of this section is to show that we have $\chi_{\mathrm{CF}}(\lambda, \kappa, \omega) \leq$ $\omega_{2}$ for $\lambda \geq \kappa \geq \omega_{2}$, provided that $\mu^{\omega}=\mu^{+}$holds for every $\mu<\lambda$ with $\operatorname{cf}(\mu)=\omega$. Moreover, if in addition $\square_{\mu}$ also holds for any $\mu$ with $\operatorname{cf}(\mu)=\omega<\mu<\lambda$, then we even have $\chi_{\mathrm{CF}}(\lambda, \kappa, \omega) \leq \omega_{1}$ whenever 
$\lambda \geq \kappa \geq \omega_{1}$. The first part will follow from a general stepping up result, whose formulation needs the following definition.

Definition 8.2. Assume that $\omega \leq \rho \leq \lambda$ are cardinals, $\mathcal{A}$ is any setsystem, and $\vec{N}=\left\langle N_{\alpha}: \alpha<\lambda\right\rangle$ is a $\lambda$-chain of elementary submodels. We say that $\vec{N} \rho$-cuts $\mathcal{A}$ iff

(16) $\mathcal{A} \in N_{1}$, moreover $\alpha<\lambda$ and $A \in \mathcal{A} \backslash N_{\alpha}$ imply $\left|A \cap N_{\alpha}\right|<\rho$.

Theorem 8.3. Let $\omega \leq \mu \leq \rho \leq \kappa \leq \lambda$ be cardinals and assume that every $(\lambda, \kappa, \mu)$-system is $\rho$-cut by a $\lambda$-chain of elementary submodels. Assume also that

(i) if $\kappa=\lambda$ then there is $\kappa^{*}<\kappa$ such that $\left[\kappa^{\prime}, \kappa^{\prime}, \mu\right] \Rightarrow \rho$ whenever $\kappa^{*} \leq \kappa^{\prime}<\kappa$ (note that in this case $\rho \leq \kappa^{*}<\kappa=\lambda$ ),

(ii) if $\kappa<\lambda$ then $\left[\lambda^{\prime}, \kappa^{\prime}, \mu\right] \Rightarrow \rho$ whenever $\kappa \leq \kappa^{\prime} \leq \lambda^{\prime}<\lambda$.

Then $[\lambda, \kappa, \mu] \Rightarrow \rho$.

Proof. Let $\mathcal{A} \subset[\lambda]^{\kappa}$ be a $(\lambda, \kappa, \mu)$-system and let $\vec{N}=\left\langle N_{\alpha}: \alpha<\lambda\right\rangle$ be a $\lambda$-chain of elementary submodels which $\rho$-cuts $\mathcal{A}$. We can assume that $\max \left(\kappa^{*}+1, \rho+1\right) \subset N_{1}$ in case $\kappa=\lambda$ and $\kappa+1 \subset N_{1}$ in case $\kappa<\lambda$. For each $\alpha<\lambda$ let

$$
\mathcal{A}_{\alpha}=\mathcal{A} \cap\left(N_{\alpha+1} \backslash N_{\alpha}\right),
$$

then $\left\langle\mathcal{A}_{\alpha}: \alpha<\lambda\right\rangle$ is a partition of $\mathcal{A}$ and $\left|\mathcal{A}_{\alpha}\right| \leq\left|N_{\alpha+1}\right|<\lambda$. We let

$$
Y_{\alpha}=\lambda \cap N_{\alpha+1} \backslash\left(N_{\alpha} \cup \bigcup \mathcal{A} \cap N_{\alpha}\right)
$$

and

$$
\mathcal{A}_{\alpha}^{\prime}=\left\{A \cap Y_{\alpha}: A \in \mathcal{A}_{\alpha}\right\}
$$

If $A \in \mathcal{A}_{\alpha}$ then $\left|A \cap N_{\alpha}\right|<\rho \leq \kappa$, hence

$$
\left|A \cap \cup\left\{Y_{\beta}: \beta<\alpha\right\}\right|<\rho,
$$

and, by definition,

$$
A \cap \cup\left\{Y_{\beta}: \beta>\alpha\right\}=\emptyset .
$$

Assume first that $\kappa=\lambda$. Then $A \in \mathcal{A}_{\alpha}$ implies

$$
\left|A \cap \bigcup\left(\mathcal{A} \cap N_{\alpha}\right)\right| \leq \mu \cdot\left|N_{\alpha}\right|<\kappa,
$$

hence, by elementarity, $\left|A \cap Y_{\alpha}\right|=\left|Y_{\alpha}\right|=\left|N_{\alpha+1}\right| \geq \kappa^{*}$. Consequently, $\mathcal{A}_{\alpha}^{\prime} \subset\left[Y_{\alpha}\right]^{\left|Y_{\alpha}\right|}$ is a $\left(\left|Y_{\alpha}\right|,\left|Y_{\alpha}\right|, \mu\right)$-system and thus, by (i), there is a function $c_{\alpha}: Y_{\alpha} \rightarrow \rho$ such that for each $A \in \mathcal{A}_{\alpha}$ we have

$$
\left|\rho \backslash I_{c_{\alpha}}\left(A \cap Y_{\alpha}\right)\right|<\rho .
$$

Assume now that $\kappa<\lambda$. Then $\cup\left(\mathcal{A} \cap N_{\alpha}\right) \subset N_{\alpha}$, and so

$$
A \cap Y_{\alpha}=A \backslash A \cap N_{\alpha} \in\left[Y_{\alpha}\right]^{\kappa} .
$$


But $\kappa \leq\left|Y_{\alpha}\right|=\left|N_{\alpha+1}\right|<\lambda$ and $\mathcal{A}_{\alpha}^{\prime} \subset\left[Y_{\alpha}\right]^{\kappa}$ is $\mu$-almost disjoint, so by (ii) there is $c_{\alpha}: Y_{\alpha} \rightarrow \rho$ such that for each $A \in \mathcal{A}_{\alpha}$ we have

$$
\left|\rho \backslash I_{c_{\alpha}}\left(A \cap Y_{\alpha}\right)\right|<\rho .
$$

Let us put (in both cases) $c=\cup\left\{c_{\alpha}: \alpha<\lambda\right\}$, then $c \in \mathcal{F}(\lambda, \rho)$. For $A \in \mathcal{A}$ pick $\alpha<\lambda$ with $A \in \mathcal{A}_{\alpha}$, then (19) implies

$$
I_{c}(A) \supset I_{c_{\alpha}}\left(A \cap Y_{\alpha}\right) \backslash c\left[A \cap \cup\left\{Y_{\beta}: \beta<\alpha\right\}\right] .
$$

But $\left|A \cap \cup\left\{Y_{\beta}: \beta<\alpha\right\}\right|<\rho$ by (18), hence either (20) or (21) implies $\left|\rho \backslash I_{c}(A)\right|<\rho$. Finally, if $\operatorname{dom}(c) \neq \lambda$ then we may extend $c$ to a full function $d: \lambda \rightarrow \rho+1$ by mapping every member of $\lambda \backslash \operatorname{dom}(c)$ to $\rho$, and then we have $\left|\varrho \backslash I_{d}(A)\right|<\rho$, which completes the proof of $[\lambda, \kappa, \mu] \Rightarrow \rho$.

Now, using the trivial relation $[\rho, \rho, \mu] \Rightarrow \rho$ and theorem 8.3 , the following result may be established by a straight-forward transfinite induction. The details are left to the reader.

Corollary 8.4. Let $\omega \leq \mu \leq \rho<\lambda$ be cardinals. If every $\left(\lambda^{\prime}, \kappa, \mu\right)$ system is $\rho$-cut by a $\lambda^{\prime}$-chain of elementary submodels whenever $\rho<$ $\lambda^{\prime} \leq \lambda$ and $\rho \leq \kappa \leq \lambda^{\prime}$ then $[\lambda, \kappa, \mu] \Rightarrow \rho$.

The following easy lemma will be used in the proof of the first result that was promised in the introductory paragraph of this section.

Lemma 8.5. Assume that $\lambda \geq \omega_{2}$ and $\mu^{\omega}=\mu^{+}$holds for each $\mu<\lambda$ with $\operatorname{cf}(\mu)=\omega$. If $\mathcal{A}$ is an $\omega$-almost disjoint set system and $X$ is any set with $|X|<\lambda$, then

$$
|\{A \in \mathcal{A}:|X \cap A|>\omega\}| \leq|X| \text {. }
$$

Proof. It obviously follows from our assumption that if $\mu<\lambda$ and $\operatorname{cf}(\mu)>\omega$ then $\mu^{\omega}=\mu$. Thus, if $\operatorname{cf}(|X|)>\omega$ then, as $\mathcal{A}$ is $\omega$-almost disjoint, we even have

$$
|\{A \in \mathcal{A}:|X \cap A| \geq \omega\}| \leq|X|^{\omega}=|X| .
$$

If, however, $\operatorname{cf}(|X|)=\omega<|X|$ then we may write $X=\cup\left\{X_{n}: n<\omega\right\}$ with $\left|X_{n}\right|<|X|$ for each $n<\omega$. But then we have

$$
\left|\left\{A \in \mathcal{A}:|X \cap A| \geq \omega_{1}\right\}\right|=\left|\left\{A \in \mathcal{A}: \exists n\left|X_{n} \cap A\right| \geq \omega_{1}\right\}\right|,
$$

and so

$$
\begin{aligned}
\left|\left\{A \in \mathcal{A}:|X \cap A| \geq \omega_{1}\right\}\right| \leq \sum_{n<\omega}\left|\left\{A \in \mathcal{A}:\left|X_{n} \cap A\right| \geq \omega\right\}\right| \leq & \\
& \leq \sum_{n<\omega}\left|X_{n}\right|^{\omega}=|X| .
\end{aligned}
$$


Theorem 8.6. Assume that $\lambda \geq \omega_{2}$ and $\mu^{\omega}=\mu^{+}$holds for each $\mu<\lambda$ with $\operatorname{cf}(\mu)=\omega$. Then $[\lambda, \kappa, \omega] \Rightarrow \omega_{2}$ whenever $\omega_{2} \leq \kappa \leq \lambda$.

Proof. By corollary 8.4, it clearly suffices to show that if $\omega_{2}<\lambda^{\prime} \leq \lambda$ and $\mathcal{A}$ is any $\omega$-almost disjoint set-system of cardinality $\lambda^{\prime}$, then $\mathcal{A}$ is $\omega_{2}$-cut by a $\lambda^{\prime}$-chain of elementary submodels.

To see this, let $\left\langle M_{\alpha}: \alpha<\lambda^{\prime}\right\rangle$ be any $\lambda^{\prime}$-chain of elementary submodels satisfying $\omega_{2} \cup\{\mathcal{A}\} \subset M_{1}$ and for every $\alpha<\lambda^{\prime}$ write $N_{\alpha}=M_{\omega \alpha}$. We claim that $\left\langle N_{\alpha}: \alpha<\lambda^{\prime}\right\rangle$, also a $\lambda^{\prime}$-chain of elementary submodels, $\omega_{2}$-cuts $\mathcal{A}$.

Indeed, assume that $\alpha<\lambda^{\prime}$ and $A \in \mathcal{A}$ with $\left|A \cap N_{\alpha}\right| \geq \omega_{2}$. Since $\omega \alpha$ is a limit ordinal, then there is $\beta<\omega \alpha$ such that $\left|A \cap M_{\beta}\right| \geq \omega_{1}$. But then $\mathcal{A}^{\prime}=\left\{A^{\prime} \in \mathcal{A}:\left|A^{\prime} \cap M_{\beta}\right| \geq \omega_{1}\right\} \in M_{\beta+1}$ and $\left|\mathcal{A}^{\prime}\right| \leq\left|M_{\beta}\right|$ by lemma 8.5, hence we have $A \in \mathcal{A}^{\prime} \subset M_{\beta+1} \subset M_{\omega \alpha}=N_{\alpha}$.

A very short alternative proof of theorem 8.6 may be obtained as follows. In [3, Theorem 6] Erdős and Hajnal proved that if $\mu^{\omega}=\mu^{+}$ holds for each $\mu<\lambda$ with $\operatorname{cf}(\mu)=\omega$ then

$$
\mathbf{M}(\lambda, \kappa, \omega) \rightarrow \mathbf{B}\left(\omega_{2}\right) \text { whenever } \omega_{1} \leq \kappa \leq \lambda .
$$

Moreover, under the same assumption, Komjáth proved in [10, Theorem 5] that

$$
\mathbf{M}\left(\lambda, \omega_{2}, \omega\right) \rightarrow \mathbf{E D} \text { for all } \lambda \geq \omega_{2} .
$$

Applying proposition 4.3 with $\mu=\omega$ and $\tau=\omega_{2}$, we may conclude that (22) and (23) together imply $[\lambda, \kappa, \omega] \Rightarrow \omega_{2}$ whenever $\lambda \geq \kappa \geq \omega_{2}$.

Actually, the above proof yields the stronger conclusion

$$
[\lambda, \kappa, \omega] \Rightarrow \omega_{1} \text { whenever } \lambda \geq \kappa \geq \omega_{1},
$$

provided that in (23) we may replace $\omega_{2}$ by $\omega_{1}$. But by $[10$, Theorem $5(\mathrm{c})$ ], this can be done if, in addition to $\mu^{\omega}=\mu^{+}$for all $\mu<\lambda$ with $\operatorname{cf}(\mu)=\omega$, we also assume $\square_{\mu}$ for each $\mu<\lambda$ with $\operatorname{cf}(\mu)=\omega<\mu$. (In fact, as it is shown in [7], the assumption of a very weak version of $\square_{\mu}$ suffices for this.) Thus we get the following result.

Theorem 8.7. Let $\lambda$ be an uncountable cardinal and assume that

(i) $\mu^{\omega}=\mu^{+}$for each cardinal $\mu<\lambda$ with $\operatorname{cf}(\mu)=\omega$,

(ii) $\square_{\mu}$ holds for each singular cardinal $\mu<\lambda$ with $\operatorname{cf}(\mu)=\omega$.

Then $[\lambda, \kappa, \omega] \Rightarrow \omega_{1}$ holds whenever $\omega_{1} \leq \kappa \leq \lambda$.

As condition (ii) of theorem 8.7 is only relevant for $\lambda>\aleph_{\omega}$, we immediately obtain the following result.

Corollary 8.8. CH and $\omega_{1} \leq \kappa \leq \lambda \leq \aleph_{\omega}$ imply $[\lambda, \kappa, \omega] \Rightarrow \omega_{1}$. 


\section{CONSistent LOWER BOUnds FOR $\chi_{\mathrm{CF}}(\lambda, \kappa, \omega)$}

In the previous section we gave (consistent) universal upper bounds for $\chi_{\mathrm{CF}}(\lambda, \kappa, \omega)$ when $\kappa \geq \omega_{2}$ and $\kappa \geq \omega_{1}$, respectively. That no such universal upper bound can be given for $\chi_{\mathrm{CF}}(\lambda, \omega, \omega)$ follows from the fact that if $\boldsymbol{\beta}(\lambda)$ holds, that is for each $\alpha \in E_{\omega}^{\lambda}$ there is an $\omega$-type subset $A_{\alpha}$ cofinal in $\alpha$ such that for every $X \in[\lambda]^{\lambda}$ we have $A_{\alpha} \subset X$ for some $\alpha \in E_{\omega}^{\lambda}$, then clearly

$$
\chi_{\mathrm{CF}}(\lambda, \omega, \omega) \geq \chi(\lambda, \omega, \omega) \geq \operatorname{cf}(\lambda) .
$$

In particular, if $\lambda$ is also regular then we have

$$
\chi_{\mathrm{CF}}(\lambda, \omega, \omega)=\chi(\lambda, \omega, \omega)=\lambda .
$$

In order to get some lower bounds for $\chi_{\mathrm{CF}}(\lambda, \kappa, \omega)$ with $\kappa>\omega$, and thus to show that the results of the previous section are sharp, we shall make use of a result in [7]. First we give some notation.

If $\lambda>\omega_{1}$ is a regular cardinal and $S \subset E_{\omega_{1}}^{\lambda}$ is stationary then we denote by $\star(S)$ the following statement:

$\star(S)$ : there is an $\omega$-almost disjoint family $\left\{A_{\alpha}: \alpha \in S\right\}$ such that $A_{\alpha}$ is a cofinal subset of $\alpha$ of order type $\omega_{1}$ for each $\alpha \in S$.

It is an immediate consequence of Fodor's pressing down theorem that such an $\left\{A_{\alpha}: \alpha \in S\right\}$ is not essentially disjoint, hence if we assume condition (i) of theorem 8.7 then (very weak) $\square_{\mu}$ must fail at some singular $\mu<\lambda$ with $\operatorname{cf}(\mu)=\omega$, in particular $\lambda>\aleph_{\omega}$. This implies that if $\star(S)$ holds then we must have some large cardinals, and in fact it was shown in [7] that the existence of a supercompact cardinal implies the consistency of GCH with $\star(S)$ for some $S \subset \aleph_{\omega+1}$.

For any set $S \subset \lambda$ we shall denote by $\boldsymbol{p}(S)$ the statement that there is a sequence $\left\{B_{\alpha}: \alpha \in S\right\}$ with $\cup B_{\alpha}=\alpha$ for each $\alpha \in S$ such that for every $X \in[\lambda]^{\lambda}$ we have $B_{\alpha} \subset X$ for some $\alpha \in S$. Then $\left\{B_{\alpha}: \alpha \in S\right\}$ is called a $\hat{\boldsymbol{s}}(S)$-sequence. Clearly, every $\diamond(S)$-sequence is a $\hat{\boldsymbol{s}}(S)$ sequence.

Theorem 9.1. Assume that $\lambda>2^{\omega}$ is a regular cardinal and we have both $\star(S)$ and $\diamond(S)$ for a stationary set $S \subset E_{\omega_{1}}^{\lambda}$. Then

(1) there is an $\omega$-almost disjoint $\mathbf{s}\left(S^{*}\right)$-sequence for some $S^{*} \subset S$, hence

$$
\chi_{\mathrm{CF}}\left(\lambda, \omega_{1}, \omega\right)=\chi\left(\lambda, \omega_{1}, \omega\right)=\lambda ;
$$

(2) for every cardinal $\kappa$ with $\omega_{2} \leq \kappa<\lambda$ we have $\omega_{2} \leq \chi_{\mathrm{CF}}(\lambda, \kappa, \omega)$.

Proof. (1) Let us fix an $\omega$-almost disjoint family $\left\{A_{\alpha}: \alpha \in S\right\}$ witnessing $\star(S)$ and a $\diamond(S)$-sequence $\left\{B_{\alpha}: \alpha \in S\right\}$. Let

$$
B_{\alpha}=\left\{b(\alpha, \gamma): \gamma<\operatorname{tp}\left(B_{\alpha}\right)\right\}
$$


be the increasing enumeration of $B_{\alpha}$.

Next, by transfinite recursion we define sets $\left\{E_{\alpha}: \alpha \in S\right\}$ as follows. Assume that $\left\{E_{\beta}: \beta \in \alpha \cap S\right\}$ has been constructed. If $\operatorname{tp}\left(B_{\alpha}\right)<\alpha$ then let $E_{\alpha}=\emptyset$. Otherwise, if $\operatorname{tp}\left(B_{\alpha}\right)=\alpha$, set

$$
E_{\alpha}=\left\{b(\alpha, \gamma): \gamma \in A_{\alpha}\right\}
$$

clearly then $E_{\alpha} \in\left[B_{\alpha}\right]^{\omega_{1}}$ is cofinal in $\alpha$.

Let us next define

$$
S^{*}=\left\{\alpha \in S:\left|E_{\alpha}\right|=\omega_{1} \wedge \forall \beta \in S \cap \alpha\left(\left|E_{\beta} \cap E_{\alpha}\right|<\omega\right)\right\},
$$

and

$$
\mathcal{E}=\left\{E_{\alpha}: \alpha \in S^{*}\right\} .
$$

Then $\mathcal{E} \subset[\lambda]^{\omega_{1}}$ is $\omega$-almost disjoint by definition and we claim that $\mathcal{E}$ is a $\boldsymbol{p}\left(S^{*}\right)$-sequence.

Indeed, let $B \in[\lambda]^{\lambda}$ and consider the club set

$$
C=\{\xi<\lambda: \operatorname{tp}(B \cap \xi)=\xi\}
$$

and the stationary set

$$
\hat{S}=\left\{\alpha \in S \cap C: B \cap \alpha=B_{\alpha}\right\} .
$$

Now, if $\alpha \in \hat{S} \cap S^{*}$ then $E_{\alpha} \subset B_{\alpha}=B \cap \alpha \subset B$, hence it suffices to show that $\hat{S} \cap S^{*} \neq \emptyset$.

Assume, on the contrary, that $\hat{S} \cap S^{*}=\emptyset$. Then for each $\alpha \in \hat{S}$, as $\operatorname{tp}\left(B_{\alpha}\right)=\alpha$, there is a $\beta<\alpha$ such that $E_{\alpha} \cap E_{\beta}$ is infinite. By Fodor's theorem and $2^{\omega}<\lambda$, there are $\beta<\alpha<\alpha^{\prime}$ and $X \in\left[E_{\beta}\right]^{\omega}$ such that $\alpha, \alpha^{\prime} \in \hat{S}$ and $X \subset E_{\alpha} \cap E_{\alpha^{\prime}}$. But $B_{\alpha}=\alpha \cap B_{\alpha^{\prime}}$, hence $b(\alpha, \gamma)=$ $b\left(\alpha^{\prime}, \gamma\right)$ for all $\gamma<\alpha$ and $b\left(\alpha^{\prime}, \gamma\right) \notin B_{\alpha}$ for $\gamma \geq \alpha$, consequently $x \in E_{\alpha} \cap E_{\alpha^{\prime}}$ implies that $x=b(\alpha, \gamma)$ for some $\gamma \in A_{\alpha} \cap A_{\alpha^{\prime}}$. This, however contradicts $\left|A_{\alpha} \cap A_{\alpha^{\prime}}\right|<\omega$, proving that $\hat{S} \cap S^{*} \neq \emptyset$ and so $\mathcal{E}$ is a $\boldsymbol{\beta}\left(S^{*}\right)$-sequence.

But then $\mathcal{E}$ is a $\left(\lambda, \omega_{1}, \omega\right)$-system for which $\chi_{\mathrm{CF}}(\mathcal{E})=\chi(\mathcal{E})=\lambda$ holds trivially, completing the proof of part (1).

(2) Having fixed $\kappa$ with $\omega_{1}<\kappa<\lambda$, we shall construct a $(\lambda, \kappa, \omega)$ system $\mathcal{F} \subset[\lambda]^{\kappa}$ such that for every function $h: \lambda \rightarrow \omega_{1}$ there is $F \in \mathcal{F}$ for which

$$
\nu \in h[F] \text { implies }\left|F \cap h^{-1}\{\nu\}\right| \geq \omega_{1} .
$$

Consider the club set $K=\{\kappa \cdot \xi: \xi<\lambda\}$ and, for every $\xi<\lambda$, let $K_{\xi}$ denote the (half-closed) interval $[\kappa \cdot \xi, \kappa \cdot(\xi+1))$. We can assume, without any loss of generality, that $S \subset K$. 
For every $\alpha \in S$ we also fix a partition of $A_{\alpha}$ into $\omega_{1}$-many disjoint uncountable pieces: $A_{\alpha}=\cup\left\{A_{\alpha, \nu}: \nu<\omega_{1}\right\}$. Finally, this time, we use $\diamond(S)$ by choosing a $\diamond(S)$-sequence $\left\{h_{\alpha}: \alpha \in S\right\}$ for the functions $h: \lambda \rightarrow \omega_{1}$.

Next, by transfinite recursion define the sets $\left\{E_{\alpha}: \alpha \in S\right\}$ as follows. Assume that $\alpha \in S$, moreover $\left\{E_{\beta}: \beta \in \alpha \cap S\right\}$ has been constructed. Let

$$
D_{\alpha}=\left\{\nu<\omega_{1}: \operatorname{tp}\left(h_{\alpha}^{-1}\{\nu\}\right)=\alpha\right\}
$$

for every $\nu \in D_{\alpha}$ let $\{b(\alpha, \nu, \eta): \eta<\alpha\}$ be the increasing enumeration of $h_{\alpha}^{-1}\{\nu\}$, and put

$$
E_{\alpha}^{\prime}=\left\{b(\alpha, \nu, \gamma): \nu \in D_{\alpha}, \gamma \in A_{\alpha, \nu}\right\} .
$$

Of course, if $D_{\alpha}=\emptyset$ then we have $E_{\alpha}^{\prime}=\emptyset$ as well, and in this case we put $E_{\alpha}=\emptyset$. If, however, $D_{\alpha} \neq \emptyset$ then for every $\nu \in D_{\alpha}$ the set $B_{\alpha, \nu}=\left\{b(\alpha, \nu, \gamma): \gamma \in A_{\alpha, \nu}\right\}$ is cofinal in $\alpha$. Thus, using that $\alpha=\kappa \cdot \xi$ for some $\xi$ with $\operatorname{cf}(\xi)=\omega_{1}<\kappa$, we can find $E_{\alpha} \subset E_{\alpha}^{\prime}$ such that (i) $\left|E_{\alpha} \cap B_{\alpha, \nu}\right|=\omega_{1}$ for each $\nu \in D_{\alpha}$, and (ii) $\left|E_{\alpha} \cap K_{\zeta}\right| \leq 1$ for every $\zeta<\lambda$.

Next, similarly as in the proof of (1), we let

$$
S^{*}=\left\{\alpha \in S:\left|E_{\alpha}\right|=\omega_{1} \wedge \forall \beta \in S \cap \alpha\left(\left|E_{\beta} \cap E_{\alpha}\right|<\omega\right)\right\},
$$

and then for any $\alpha=\kappa \cdot \xi \in S^{*}$ we define

$$
F_{\alpha}=E_{\alpha} \cup K_{\xi}=E_{\alpha} \cup[\alpha, \alpha+\kappa) \text {, and } \mathcal{F}=\left\{F_{\alpha}: \alpha \in S^{*}\right\} \text {. }
$$

Clearly, $\mathcal{F} \subset[\lambda]^{\kappa}$ and $\mathcal{F}$ is $\omega$-almost disjoint because, by (ii), we have $\left|F_{\alpha} \cap F_{\beta}\right| \leq\left|E_{\alpha} \cap E_{\beta}\right|+1$ for any $\{\alpha, \beta\} \in\left[S^{*}\right]^{2}$.

Now, consider any map $h: \lambda \rightarrow \omega_{1}$ and let

$$
D=\left\{\nu<\omega_{1}:\left|h^{-1}\{\nu\}\right|=\lambda\right\}
$$

then $D \neq \emptyset$. For every $\nu \in D$ put

$$
C_{\nu}=\left\{\xi<\lambda: \operatorname{tp}\left(\xi \cap\left(h^{-1}\{\nu\}\right)\right)=\xi\right\}
$$

and

$$
C=\cap\left\{\left(C_{\nu}: \nu \in D\right\}\right.
$$

then $C$ is a club set.

We have $\eta=\sup \left(h^{-1}\left[\omega_{1} \backslash D\right]\right)<\lambda$ because $\lambda>\omega_{1}$ is regular. Let $T=S \cap C \backslash \eta$, then $h[T] \subset D$,

$$
\hat{S}=\left\{\alpha \in T: h \uparrow \alpha=h_{\alpha}\right\}
$$

is stationary, and if $\alpha \in \hat{S}$ then $D_{\alpha}=D$.

Note that if $\alpha \in \hat{S} \cap S^{*}$ then $h\left[F_{\alpha}\right]=h\left[E_{\alpha}\right]=D_{\alpha}=D$ and, by our construction,

$$
\left|h^{-1}\{\nu\} \cap E_{\alpha}\right|=\omega_{1}
$$


for each $\nu \in D$, hence $F_{\alpha} \in \mathcal{F}$ witnesses (24). Thus, to prove part (2), it again suffices to show that $\hat{S} \cap S^{*} \neq \emptyset$.

Assume, on the contrary, that $\hat{S} \cap S^{*}=\emptyset$. Since $D_{\alpha}=D \neq \emptyset$ for every $\alpha \in \hat{S} \subset C$ this would imply that for every $\alpha \in \hat{S}$ there exists $\beta<\alpha$ for which $E_{\alpha} \cap E_{\beta}$ is infinite. But then, in the same way as in the proof of (1), we could conclude that there is a pair $\left\{\alpha, \alpha^{\prime}\right\} \in[\hat{S}]^{2}$ with $\alpha<\alpha^{\prime}$ such that $E_{\alpha} \cap E_{\alpha^{\prime}}$ is infinite. Using that $h_{\alpha}=h_{\alpha^{\prime}} \uparrow \alpha$ and hence $h_{\alpha}^{-1}\{\nu\}$ is an initial segment of $h_{\alpha^{\prime}}^{-1}\{\nu\}$, this would imply that $A_{\alpha} \cap A_{\alpha^{\prime}}$ is also infinite, a contradiction.

As we noted above, it was shown in [7] that the existence of a supercompact cardinal implies the consistency of GCH with $\star(S)$ for some $S \subset E_{\omega_{1}}^{\aleph_{\omega+1}}$. This, together with theorem 9.1, immediately yields the following result which shows that the results of the previous section are sharp, even under GCH.

Corollary 9.2. If it is consistent that there is a supercompact cardinal then it is also consistent that GCH holds and

(1) $\chi\left(\aleph_{\omega+1}, \omega_{1}, \omega\right)=\chi_{\mathrm{CF}}\left(\aleph_{\omega+1}, \omega_{1}, \omega\right)=\aleph_{\omega+1}$,

(2) $\chi_{\mathrm{CF}}\left(\aleph_{\omega+1}, \omega_{n}, \omega\right)=\omega_{2}$ for $2 \leq n \leq \omega$.

We conclude this section with a (somewhat surprising) result showing that consistently, e.g. under $\mathrm{GCH}$, the relation $\chi\left(\lambda, \omega_{1}, \omega\right) \leq \omega_{1}$, hence $\chi_{\mathrm{CF}}\left(\lambda, \omega_{1}, \omega\right) \leq \omega_{1}$ as well, implies $\mathbf{M}\left(\lambda, \omega_{1}, \omega\right) \rightarrow \mathbf{E D}$.

Theorem 9.3. Let $\lambda$ be an uncountable cardinal and assume that

(i) $\mu^{\omega}=\mu^{+}$for any $\mu<\lambda$ with $\operatorname{cf}(\mu)=\omega$,

(ii) if $\omega<\mu<\lambda$ with $\operatorname{cf}(\mu)=\omega$ then $\diamond(S)$ holds for every stationary set $S \subset E_{\omega_{1}}^{\mu^{+}}$.

Then $\chi\left(\lambda, \omega_{1}, \omega\right) \leq \omega_{1}$ implies $\mathbf{M}\left(\lambda, \omega_{1}, \omega\right) \rightarrow \mathbf{E D}$.

Proof. We shall prove this by induction on $\lambda$. It is trivially true for $\lambda=\omega_{1}$, hence we can assume $\lambda>\omega_{1}$ and that it holds for all $\lambda^{\prime}<\lambda$.

We shall make use of the following obvious corollary of our assumption (i): If $X$ is any set with $|X| \leq \lambda$ and $\mathcal{F} \subset[X]^{\omega_{1}}$ is $\omega$-almost disjoint then $|\mathcal{F}| \leq|X|$. In fact, this follows from the following consequence of (i): $\mu^{\omega}=\mu$ if $\mu \leq \lambda$ with $\operatorname{cf}(\mu)>\omega$.

Now, let $\mathcal{A} \subset[\lambda]^{\omega_{1}}$ be an $\omega$-almost disjoint set-system, we have to show that $\mathcal{A}$ is essentially disjoint.

Case 1: $\lambda$ is a limit cardinal or $\lambda=\mu^{+}$for some $\mu$ with $\operatorname{cf}(\mu)>\omega$.

Condition (i) implies $\nu^{\omega}<\lambda$ for any $\nu<\lambda$, hence we can find a $\lambda$-chain $\left\langle M_{\alpha}: \alpha<\lambda\right\rangle$ of elementary submodels with $\mathcal{A} \in M_{1}$ and 
$\omega_{1} \subset M_{1}$ such that $\left[M_{\alpha}\right]^{\omega} \subset M_{\alpha+1}$ for each $\alpha<\lambda$. Let us put $N_{\alpha}=M_{\omega \cdot \alpha}$ for $\alpha<\lambda$, then $\mathcal{A}$ is $\omega_{1}$-cut by the $\lambda$-chain $\left\langle N_{\alpha}: \alpha<\lambda\right\rangle$.

Indeed, if $\left|A \cap N_{\alpha}\right|=\left|A \cap M_{\omega \cdot \alpha}\right|=\omega_{1}$ then there is a $\beta<\omega \cdot \alpha$ such that $\left|A \cap M_{\beta}\right| \geq \omega$. Since $\mathcal{A}$ is $\omega$-almost disjoint and $\left[M_{\beta}\right]^{\omega} \subset M_{\beta+1}$ then we have $A \in M_{\beta+1} \subset M_{\omega \cdot \alpha}=N_{\alpha}$.

For $\alpha<\lambda$ let

$$
\mathcal{A}_{\alpha}=\mathcal{A} \cap\left(N_{\alpha+1} \backslash N_{\alpha}\right),
$$

then $\left|\mathcal{A}_{\alpha}\right| \leq\left|N_{\alpha+1}\right|<\lambda$. By this and the inductive hypothesis there is a function $F_{\alpha}: \mathcal{A}_{\alpha} \rightarrow[\lambda]^{\omega}$ such that $A \cap N_{\alpha} \subset F_{\alpha}(A)$ for all $A \in \mathcal{A}_{\alpha}$ and the family

$$
\left\{A \backslash F_{\alpha}(A): A \in \mathcal{A}_{\alpha}\right\}
$$

is disjoint. Now, it is easy to check that the function

$$
F=\cup_{\alpha<\lambda} F_{\alpha}: \mathcal{A} \rightarrow[\lambda]^{\omega}
$$

witnesses the essential disjointness of $\mathcal{A}$.

Case 2: $\lambda=\tau^{+}$for some singular cardinal $\tau$ with $\operatorname{cf}(\tau)=\omega$.

For any $A \in \mathcal{A}$ let

$$
L(A)=\left\{\alpha<\lambda: \operatorname{cf}(\alpha)=\omega_{1} \text { and } \alpha=\sup A \cap \alpha\right\} .
$$

Clearly, then $1 \leq|L(A)| \leq \omega_{1}$. We claim that the set

$$
S=\cup\{L(A): A \in \mathcal{A}\}
$$

is non-stationary in $\lambda$.

Indeed, by definition, for each $A \in \mathcal{A}$ we may find a family of pairwise disjoint sets

$$
\{B(A, \alpha): \alpha \in L(A)\} \subset[A]^{\omega_{1}}
$$

such that $\sup (B(A, \alpha))=\alpha$ and $\operatorname{tp}\left(B(A, \alpha)=\omega_{1}\right.$. So, if $S$ were stationary then the $\omega$-almost disjoint family

$$
\mathcal{B}=\{B(A, \alpha): A \in \mathcal{A}, \alpha \in L(A)\}
$$

would witness $\star(S)$. But then, by condition (ii) and part (1) of theorem 9.1 , we would have $\chi\left(\lambda, \omega_{1}, \omega\right)=\lambda>\omega_{1}$, a contradiction. So there is a club $E \subset \lambda$ such that

$$
E \cap \cup\{L(A): A \in \mathcal{A}\}=\emptyset .
$$

It follows from our introductory remark that if $\delta<\lambda$ then

$$
\left|\left\{A \in \mathcal{A}:|A \cap \delta|=\omega_{1}\right\}\right| \leq \delta<\lambda,
$$

hence the following set $D$ is club in $\lambda$ :

$$
D=\left\{\zeta<\lambda: \forall \delta<\zeta \forall A \in \mathcal{A}\left(|A \cap \delta|=\omega_{1} \text { implies } A \subset \zeta\right)\right\} .
$$


Let $C=E \cap D$ and $C=\left\{\gamma_{\nu}: \nu<\lambda\right\}$ be the increasing enumeration of $C$.

For any $A \in \mathcal{A}$ let

$$
\nu_{A}=\min \left\{\nu<\lambda:\left|A \cap \gamma_{\nu}\right|=\omega_{1}\right\} .
$$

Then $C \subset E$ implies that $\nu_{A}$ can not be a limit ordinal, hence $\nu_{A}=$ $\eta_{A}+1$. This and the definition of $D$ imply

$$
\left|A \cap \gamma_{\eta_{A}}\right| \leq \omega \text { and } A \subset \gamma_{\eta_{A}+1}
$$

Let us put $\mathcal{A}_{\eta}=\left\{A \in \mathcal{A}: \eta_{A}=\eta\right\}$ for any $\eta<\lambda$, then $\left|\mathcal{A}_{\eta}\right| \leq$ $\gamma_{\eta+1}<\lambda$. By the inductive hypothesis, for each $\eta<\lambda$ there is a function $F_{\eta}: \mathcal{A}_{\eta} \rightarrow[\lambda]^{\omega}$ such that $A \cap \gamma_{\eta} \subset F_{\eta}(A)$ for any $A \in \mathcal{A}_{\eta}$ and the family

$$
\left\{A \backslash F_{\eta}(A): A \in \mathcal{A}_{\eta}\right\}
$$

is disjoint. Now, it is again easy to check that the function

$$
F=\cup_{\eta<\lambda} F_{\eta}: \mathcal{A} \rightarrow[\lambda]^{\omega}
$$

witnesses the essential disjointness of $\mathcal{A}$.

Let us remark that, by a recent result of Shelah from [14, if $\omega=$ $\operatorname{cf}(\mu)<\mu$ and $2^{\mu}=\mu^{+}$then $\diamond(S)$ holds for every stationary set $S \subset$ $E_{\omega_{1}}^{\mu^{+}}$. Consequently, conditions (i) and (ii) of theorem 9.3 together are equivalent with the following single statement: For all $\mu<\lambda$ with $\operatorname{cf}(\mu)=\omega$ we have $2^{\mu}=\mu^{+}$.

If $\mathcal{A}$ is an essentially disjoint $\left(\lambda, \omega_{1}, \omega\right)$-system then, trivially, we have $\chi(\mathcal{A})=2$, moreover there is a coloring $f: \cup \mathcal{A} \rightarrow \omega_{1}$ that satisfies $\left|\omega_{1} \backslash I_{f}(A)\right|<\omega_{1}$ for all $A \in \mathcal{A}$. Consequently, from theorem 9.3 we immediately obtain the following result.

Corollary 9.4. Under the assumptions of theorem 9.3, in particular under GCH, the following five statements are equivalent for an uncountable cardinal $\lambda$ :

1) $\left[\lambda, \omega_{1}, \omega\right] \Rightarrow \omega_{1}$

2) $\chi_{\mathrm{CF}}\left(\lambda, \omega_{1}, \omega\right) \leq \omega_{1}$,

3) $\chi\left(\lambda, \omega_{1}, \omega\right) \leq \omega_{1}$,

4) $\chi\left(\lambda, \omega_{1}, \omega\right)=2$,

5) $\mathbf{M}\left(\lambda, \omega_{1}, \omega\right) \rightarrow \mathbf{E D}$. 


$$
\text { 10. ON } \chi_{\mathrm{CF}}\left(\omega_{1}, \omega_{1}, \omega\right) \text { AND } \chi_{\mathrm{CF}}\left(\omega_{1}, \omega, \omega\right)
$$

Our previous results give no help in deciding the exact values of $\chi_{\mathrm{CF}}\left(\omega_{1}, \omega_{1}, \omega\right)$ and $\chi_{\mathrm{CF}}\left(\omega_{1}, \omega, \omega\right)$, except proposition 8.1 which implies that both are equal to either $\omega$ or $\omega_{1}$. We shall show below that actually both equal $\omega_{1}$ under $\mathrm{CH}$ and both equal $\omega$ under $M A_{\aleph_{1}}$. We also remark that, as any $\left(\omega_{1}, \omega_{1}, \omega\right)$-system clearly has an $\omega$-witness, we have

$$
\omega \leq \chi_{\mathrm{CF}}\left(\omega_{1}, \omega_{1}, \omega\right) \leq \chi_{\mathrm{CF}}\left(\omega_{1}, \omega, \omega\right) \leq \omega_{1}
$$

in ZFC. However, we do not know if their equality is provable in ZFC.

That $\mathrm{CH}$ implies $\chi_{\mathrm{CF}}\left(\omega_{1}, \omega, \omega\right)=\omega_{1}$ is an immediate consequence of the following ZFC result of Komjáth [9].

\section{Theorem 10.1.}

$$
\chi\left(2^{\omega}, \omega, \omega\right)=2^{\omega} .
$$

Before giving our proof that $\mathrm{CH}$ also implies $\chi_{\mathrm{CF}}\left(\omega_{1}, \omega_{1}, \omega\right)=\omega_{1}$, we need a preparatory lemma.

Lemma 10.2. Let $\mathcal{A} \subset\left[\omega_{1}\right]^{\omega_{1}}$ be $\omega$-almost disjoint and $\mathcal{I}(\mathcal{A})$ be the ideal generated by $\mathcal{A}$, that is, $X \in \mathcal{I}(\mathcal{A})$ iff there is $\mathcal{B} \in[\mathcal{A}]^{<\omega}$ with $X \subset \cup \mathcal{B}$. Then, for any $X \subset \omega_{1}, X \cap \alpha \in \mathcal{I}(\mathcal{A})$ for all $\alpha<\omega_{1}$ implies $X \in \mathcal{I}(\mathcal{A})$.

Proof. For each $\alpha<\omega_{1}$ we may pick a $\subset$-minimal $\mathcal{B}_{\alpha} \in[\mathcal{A}]^{<\omega}$ such that $X \cap \alpha \subset^{*} \cup \mathcal{B}_{\alpha}$, i.e. $\left|X \cap \alpha \backslash \cup \mathcal{B}_{\alpha}\right|<\omega$. There is $I \in\left[\omega_{1}\right]^{\omega_{1}}$ for which $\left\{\mathcal{B}_{\alpha}: \alpha \in I\right\}$ forms a $\Delta$-system with kernel $\mathcal{B}$. We claim that $\mathcal{B}_{\alpha}=\mathcal{B}$ for all $\alpha \in I$. Then we are done because this implies $X \subset^{*} \cup \mathcal{B}$ and hence $X \in \mathcal{I}(\mathcal{A})$ by $X \subset \cup \mathcal{A}$.

So assume, on the contrary, that $\alpha \in I$ and $A \in \mathcal{B}_{\alpha} \backslash \mathcal{B}$. By the $\subset$-minimality of $\mathcal{B}_{\alpha}$ then

$$
Y=A \cap(X \cap \alpha \backslash \cup \mathcal{B})
$$

must be infinite. But, for any $\beta \in I$ with $\beta>\alpha$, if $B \in \mathcal{B}_{\beta} \backslash \mathcal{B}$ then $|B \cap Y| \leq|B \cap A|<\omega$, contradicting

$$
Y \subset X \cap \beta \backslash \cup \mathcal{B} \subset^{*} \cup\left(\mathcal{B}_{\beta} \backslash \mathcal{B}\right)
$$

Theorem 10.3. $C H$ implies

$$
\chi_{\mathrm{CF}}\left(\omega_{1}, \omega_{1}, \omega\right)=\chi_{\mathrm{CF}}\left(\omega_{1}, \omega, \omega\right)=\omega_{1} .
$$

Proof. By induction on $\alpha$, we shall construct an $\omega$-almost disjoint family $\mathcal{A}=\left\{A_{\alpha}: \alpha<\omega_{1}\right\} \subset\left[\omega_{1}\right]^{\omega_{1}}$ such that for any coloring $h: \omega_{1} \rightarrow \omega$ there is $A_{\alpha} \in \mathcal{A}$ satisfying

$$
\forall n \in h\left[A_{\alpha}\right]\left(\left|h^{-1}\{n\} \cap A_{\alpha}\right|=\omega\right) .
$$


To start with, using $\mathrm{CH}$, let

- $\left\{T_{\alpha}: \alpha<\omega_{1}\right\}$ be a partition of $\omega_{1}$ into uncountable sets such that $T_{\alpha} \subset \omega_{1} \backslash \alpha$ for every $\alpha<\omega_{1}$;

- $\left\{S_{\alpha}: \alpha<\omega_{1}\right\}$ be an enumeration of $\left[\omega_{1}\right]^{\omega}$.

Assume that $\left\{A_{\beta}: \beta<\alpha\right\}$ has been constructed and we have $\alpha \in T_{\gamma}$. For any subset $a \subset \alpha$ we write $A[a]=\cup\left\{A_{\beta}: \beta \in a\right\}$, in particular, $A[\xi]=\cup_{\eta<\xi} A_{\eta}$. Consider the set

$$
H_{\alpha}=\left\{\beta<\alpha: S_{\beta} \subset \alpha \backslash A[\gamma] \text { and } \forall a \in[\alpha]^{<\omega}\left|S_{\beta} \backslash A[a]\right|=\omega\right\} .
$$

We can choose $B_{\alpha} \subset \alpha \backslash A[\gamma]$ such that

(1) $\left|B_{\alpha} \cap A_{\beta}\right|<\omega$ for each $\beta<\alpha$,

(2) $\left|B_{\alpha} \cap S_{\beta}\right|=\omega$ whenever $\beta \in H_{\alpha}$.

Indeed, if $H_{\alpha}=\emptyset$ then $B_{\alpha}=\emptyset$ works, and otherwise $B_{\alpha}$ can be obtained by a simple recursive construction. Finally, let us put $A_{\alpha}=B_{\alpha} \cup T_{\alpha}$. Note that, by definition, $A_{\beta} \cap A_{\alpha}=A_{\beta} \cap B_{\alpha}$ is finite for every $\beta<\alpha$.

Let $A=A\left[\omega_{1}\right]=\cup \mathcal{A}$ and consider any coloring $h: A \rightarrow \omega$. We set

$$
I=\left\{n \in \omega: \exists \delta<\omega_{1}\left(h^{-1}\{n\} \subset A[\delta]\right)\right\}
$$

and $K=\omega \backslash I$. We may then find $\gamma<\omega_{1}$ such that $h^{-1}(I) \subset A[\gamma]$.

For any $n \in K$ consider the set

$$
X_{n}=h^{-1}\{n\} \backslash A[\gamma],
$$

then obviously $X_{n} \notin \mathcal{I}(\mathcal{A})$. Thus, by lemma 10.2, there is $\alpha_{n}<\omega_{1}$ such that $X_{n} \cap \alpha_{n} \notin \mathcal{I}(\mathcal{A})$ as well. For each $n \in K$ pick $\beta_{n}<\omega_{1}$ with $S_{\beta_{n}}=X_{n} \cap \alpha_{n}$ and choose $\alpha \in T_{\gamma}$ such that $\alpha>\sup \left\{\beta_{n}: n \in K\right\}$.

Clearly, then $\left\{\beta_{n}: n \in K\right\} \subset H_{\alpha}$, hence

$$
B_{\alpha} \cap h^{-1}\{n\} \supset B_{\alpha} \cap\left(X_{n} \cap \alpha_{n}\right)=B_{\alpha} \cap S_{\beta_{n}}
$$

is infinite for every $n \in K$. If, however, $n \in I$ then $h^{-1}\{n\} \subset A[\gamma]$, and so $B_{\alpha} \cap A[\gamma]=A_{\alpha} \cap A[\gamma]=\emptyset$ implies $h^{-1}\{n\} \cap A_{\alpha}=\emptyset$. Thus $A_{\alpha}$ witnesses (31).

Now we turn to our other promised result, namely that $M A_{\omega_{1}}$ implies

$$
\chi_{\mathrm{CF}}\left(\omega_{1}, \omega_{1}, \omega\right)=\chi_{\mathrm{CF}}\left(\omega_{1}, \omega, \omega\right)=\omega .
$$

In fact, we prove the following stronger theorem.

Theorem 10.4. If $M A_{\omega_{1}}$ holds then

(1) $\left[\omega_{1}, \omega, \omega\right] \Rightarrow \omega$,

(2) $\left[\omega_{1}, \omega_{1}, \omega\right] \Rightarrow \omega$. 
Proof. Let us start by noting that (2) follows from (1) because every $\left(\omega_{1}, \omega_{1}, \omega\right)$-system admits an $\omega$-witness.

Now, to prove $(1)$, let us consider any $\left(\omega_{1}, \omega, \omega\right)$-system $\mathcal{A}=\left\{A_{\alpha}\right.$ : $\left.\alpha<\omega_{1}\right\} \subset\left[\omega_{1}\right]^{\omega}$. We then define a poset $\mathcal{P}=\langle P, \preceq\rangle$ as follows. Let $P=F n\left(\omega_{1}, \omega\right) \times\left[\omega_{1}\right]^{<\omega}$ and for $\langle f, I\rangle,\langle g, J\rangle \in P$ put $\langle g, J\rangle \preceq\langle f, I\rangle$ iff $g \supset f, J \supset I$, and for all $\alpha \in I$ we have

(i) $(g \backslash f) \uparrow A_{\alpha}$ is $1-1$, and

(ii) $(g \backslash f)\left[A_{\alpha}\right] \cap f\left[A_{\alpha}\right]=\emptyset$.

It is easy to check that $\preceq$ is indeed a partial order on $P$.

We next show that $\mathcal{P}$ is CCC. To see this, consider first two members of $P$, say $p=\langle f, I\rangle$ and $q=\langle g, J\rangle$, such that the following conditions hold with $D=\operatorname{dom} f$ and $E=\operatorname{dom} g$ :

(a) $f \uparrow D \cap E=g \uparrow D \cap E$, i.e. $f$ and $g$ are compatible functions;

(b) $A[I] \cap(E \backslash D)=\emptyset=A[J] \cap(D \backslash E)$.

(Here, as in the proof of theorem 10.3, $A[x]=\cup\left\{A_{\alpha}: \alpha \in x\right\}$.) Then, trivially, $r=\langle f \cup g, I \cup J\rangle \in P$ and $r \preceq p, q$. Indeed, for instance, $r \preceq p$ because $(g \backslash f) \uparrow A_{\alpha}=\emptyset$ for each $\alpha \in I$. Thus, to show that $\mathcal{P}$ is CCC, it will suffice to prove that among any $\omega_{1}$ members of $P$ there are two that satisfy (a) and (b).

So let $\left\{p_{\nu}: \nu<\omega_{1}\right\} \subset P$ with $p_{\nu}=\left\langle f_{\nu}, I_{\nu}\right\rangle$. Using standard $\Delta$ system and counting arguments we can assume the following:

1) $\left\{\operatorname{dom}\left(f_{\nu}\right): \nu<\omega_{1}\right\}$ forms a $\Delta$-system with kernel $D$ and we have $D<D_{\nu}<D_{\mu}$ for $\nu<\mu<\omega_{1}$, where $D_{\nu}=\operatorname{dom}\left(f_{\nu}\right) \backslash D$.

2) $f_{\nu} \uparrow D=f$ and $\left|D_{\nu}\right|=n$ for all $\nu<\omega_{1}$.

3) $\left\{I_{\nu}: \nu<\omega_{1}\right\}$ forms a $\Delta$-system with kernel $I$ and $I<J_{\nu}<J_{\mu}$ for $\nu<\mu<\omega_{1}$, where $J_{\nu}=I_{\nu} \backslash I$. Moreover, $\left|J_{\nu}\right|=m$ for all $\nu<\omega_{1}$.

4) $A[I]<D_{0}$ and $A\left[I_{\nu}\right]<D_{\mu}$ whenever $\nu<\mu<\omega_{1}$.

Claim 10.4.1. If $N \in\left[\omega_{1}\right]^{\omega}$ and $M \in\left[\omega_{1}\right]^{n \cdot m+1}$ satisfy $N<M$ then there are $\nu \in N$ and $\mu \in M$ such that $D_{\nu} \cap A\left[J_{\mu}\right]=\emptyset$.

Proof of the claim. Let $\mathcal{U}$ be a non-principal ultrafilter on $N$. Write $D_{\nu}=\left\{\delta_{\nu, i}: i<n\right\}$ and $J_{\mu}=\left\{\alpha_{\mu, j}: j<m\right\}$.

Assume, on the contrary, that for any $\nu \in N$ and $\mu \in M$ there are $i<n$ and $j<m$ such that $\delta_{\nu, i} \in A_{\alpha_{\mu, j}}$. This implies that, for any fixed $\mu \in M$, there is a pair $\langle i, j\rangle \in n \times m$ for which

$$
V_{\mu}^{i, j}=\left\{\nu \in N: \delta_{\nu, i} \in A_{\alpha_{\mu, j}}\right\} \in \mathcal{U} .
$$

Then, as $|M|>n \cdot m$, there are two distinct $\mu, \mu^{\prime} \in M$ and a pair $\langle i, j\rangle \in n \times m$ such that both $V_{\mu}^{i, j} \in \mathcal{U}$ and $V_{\mu^{\prime}}^{i, j} \in \mathcal{U}$ and hence 
$V_{\mu}^{i, j} \cap V_{\mu^{\prime}}^{i, j} \in \mathcal{U}$ is infinite. This, however, would imply that

$$
A_{\alpha_{\mu}, j} \cap A_{\alpha_{\mu^{\prime}, j}} \supset\left\{\delta_{\nu, i}: \nu \in V_{\mu}^{i, j} \cap V_{\mu^{\prime}}^{i, j}\right\}
$$

is also infinite, a contradiction.

But if $\nu, \mu$ are as in claim 10.4.1, then clearly (a) and (b) are satisfied for $p_{\nu}$ and $p_{\mu}$, and hence they are compatible. This completes the proof that $\mathcal{P}$ is CCC.

Let us now consider, for every $\alpha<\omega_{1}$ and $n<\omega$, the sets

$$
D_{\alpha}=\{\langle f, I\rangle \in P: \alpha \in \operatorname{dom}(f)\}
$$

and

$$
E_{\alpha}^{n}=\left\{\langle f, I\rangle \in P: \alpha \in I \text { and } n \in f\left[A_{\alpha}\right]\right\} .
$$

It is easy to check that all these sets are dense in $\mathcal{P}$, let us only do it for the $E_{\alpha}^{n}$. Indeed, any $\langle f, I\rangle \in P$ is extended by $\langle f, I \cup\{\alpha\}\rangle$, so we may assume that $\alpha \in I$, to begin with. Now, if $n \notin \operatorname{ran}(f)$ then pick first $\gamma \in A_{\alpha} \backslash A[I \backslash\{\alpha\}]$. Obviously, we have then $\langle f \cup\{\langle\gamma, n\rangle\}, I\rangle \preceq\langle f, I\rangle$ and $\langle f \cup\{\langle\gamma, n\rangle\}, I\rangle \in E_{\alpha}^{n}$.

By $M A_{\omega_{1}}$ there is a filter $\mathcal{G}$ in $\mathcal{P}$ that meets all the dense sets $D_{\alpha}$ and $E_{\alpha}^{n}$. Let us put

$$
F=\cup\{f:\langle f, I\rangle \in \mathcal{G}\} .
$$

Then $F: \omega_{1} \rightarrow \omega$ because $\mathcal{G}$ meets every $D_{\alpha}$ and we claim that $I_{F}\left(A_{\alpha}\right)={ }^{*} \omega$ for each $\alpha<\omega_{1}$. Indeed, $\mathcal{G} \cap E_{\alpha}^{n} \neq \emptyset$ for all $n<\omega$ implies $F\left[A_{\alpha}\right]=\omega$. Moreover, there is some $\langle f, I\rangle \in \mathcal{G}$ with $\alpha \in I$, consequently, by the definition of $\preceq$, we clearly have $I_{F}\left(A_{\alpha}\right) \supset \omega \backslash f\left[A_{\alpha}\right]$.

Problem 10.1. Is $\chi_{\mathrm{CF}}\left(\omega_{1}, \omega_{1}, \omega\right)=\chi_{\mathrm{CF}}\left(\omega_{1}, \omega, \omega\right)$ provable in $Z F C$ ?

Recall that "stick" is the following combinatorial statement, a common weakening of $\mathrm{CH}$ and $\boldsymbol{\beta}=\boldsymbol{\beta}\left(\omega_{1}\right)$ : There is a family $\mathcal{A} \subset\left[\omega_{1}\right]^{\omega}$ such that $|\mathcal{A}|=\omega_{1}$ and for every uncountable set $S \subset \omega_{1}$ we have an $A \in \mathcal{A}$ with $A \subset S$. We know that stick implies $\chi_{\mathrm{CF}}\left(\omega_{1}, \omega, \omega\right)=\omega_{1}$.

Problem 10.2. Does stick imply $\chi_{\mathrm{CF}}\left(\omega_{1}, \omega_{1}, \omega\right)=\omega_{1}$ ?

Problem 10.3. Is $\chi_{\mathrm{CF}}\left(2^{\omega}, 2^{\omega}, \omega\right)=2^{\omega}$ provable in $Z F C$ ? 


\section{REFERENCES}

[1] P. Cheilaris: Conflict-Free Coloring, PhD thesis, City University of New York, 2008.

[2] P. Erdős, F. Galvin and A. Hajnal: On set-systems having large chromatic number and not containing prescribed subsystems, Infinite and finite sets (Colloq., Keszthely, 1973; dedicated to P. Erdős on his 60th birthday), Vol. I; Colloq. Math. Soc. János Bolyai, Vol. 10 , pp. 425-513, North-Holland, Amsterdam, 1975

[3] P. Erdős and A. Hajnal: On a property of families of sets, Acta Math. Acad. Sci. Hung., 12,(1961) 87-124

[4] P. Erdős and A. Hajnal: On the chromatic number of graphs and set-systems, Acta Math. Acad. Sci. Hung., 17,(1966) 159-229

[5] P. Erdős, A. Hajnal and B. Rothchild: On chromatic numbers of graphs and set-systems, Proceedings of the 1971 Cambridge Summer School, Springer Lecture Notes in Math., no. 337, (1971), pp. 531-538.

[6] G. Even, Z. Lotker, D. Ron, and S. Smorodinsky: SIAM J. Comput., 33, (2003) 94-136.

[7] A.Hajnal, I. Juhász and S. Shelah: Splitting strongly almost disjoint families, Trans. Amer. Math. Soc., 295, (1986) 369-387

[8] A.Hajnal, I. Juhász and S. Shelah: Strongly almost disjoint families, revisited, Fund. Math., 163, (2000) 13-23

[9] P. Komjáth: Dense systems of almost-disjoint sets. Finite and infinite sets, (Eger, 1981), 527-536, Coll Math Soc. J. Bolyai, 10, 1984.

[10] P. Komjáth: Families close to disjoint ones, Acta Math. Hungar. 43 (1984), 199-207

[11] K. Kunen: Set Theory, North-Holland, New York, 1980.

[12] E. W. Miller: On a property of families of sets, Comptes Rendus Varsovie, 30, (1937) 31-38

[13] J. Pach and G. Tardos: Conflict-free colourings of graphs and hypergraphs, Combin. Probab. Comput. 18 (2009), no. 5, 819-834.

[14] S. Shelah: Diamonds, preprint of paper $\sharp 922$, arXiv:0711.3030

Alfréd Rényi Institute of Mathematics, Budapest, Hungary

E-mail address: ahajnal@renyi.hu

Alfréd Rényi Institute of Mathematics, Budapest, Hungary

E-mail address: juhasz@renyi.hu

Alfréd Rényi Institute of Mathematics, Budapest, Hungary

E-mail address: soukup@renyi.hu

$U R L:$ http://www.renyi.hu/soukup

EÖTVÖs UNIVERSITY OF BUDAPEST

E-mail address: zoli@renyi.hu 